\title{
Integrable lattice models and holography
}

\section{Meer Ashwinkumar}

Department of Physics, National University of Singapore, 2 Science Drive 3, 117551 Singapore

E-mail: meerashwinkumar@u.nus.edu

Abstract: We study four-dimensional Chern-Simons theory on $D \times \mathbb{C}$ (where $D$ is a disk), which is understood to describe rational solutions of the Yang-Baxter equation from the work of Costello, Witten and Yamazaki. We find that the theory is dual to a boundary theory, that is a three-dimensional analogue of the two-dimensional chiral WZW model. This boundary theory gives rise to a current algebra that turns out to be an "analyticallycontinued" toroidal Lie algebra. In addition, we show how certain bulk correlation functions of two and three Wilson lines can be captured by boundary correlation functions of local operators in the three-dimensional WZW model. In particular, we reproduce the leading and subleading nontrivial contributions to the rational $R$-matrix purely from the boundary theory.

Keywords: Chern-Simons Theories, Lattice Integrable Models

ARXIV EPRINT: 2003.08931

Dedicated to my mother, Dr. Indra. 


\section{Contents}

1 Introduction 1

2 3d "Chiral" WZW model 2

2.1 Current algebra via canonical quantization 4

3 Wilson lines and boundary local operators $\quad 8$

3.1 Non-perpendicular Wilson lines 11

$\begin{array}{lll}3.2 & \text { Crossed Wilson lines at order } \hbar^{2} & 15\end{array}$

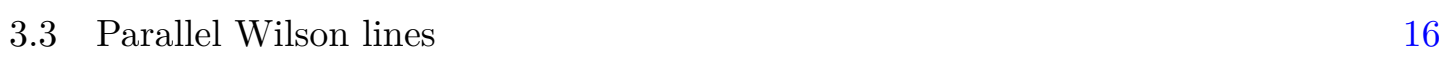

$\begin{array}{lll}3.4 & \text { Three Wilson lines } & 18\end{array}$

\section{Introduction}

A relatively novel approach to the study of integrable lattice models underlaid by the Yang-Baxter equation with spectral parameters is that of four-dimensional Chern-Simons theory, first proposed by Costello [1, 2], and subsequently studied by Costello, Witten and Yamazaki [3-5] in depth.

The theory is defined by the path integral involving the classical action

$$
S=\frac{1}{2 \pi \hbar} \int_{\Sigma \times C} \omega \wedge \operatorname{Tr}\left(\mathcal{A} \wedge d \mathcal{A}+\frac{2}{3} \mathcal{A} \wedge \mathcal{A} \wedge \mathcal{A}\right)
$$

where $\mathcal{A}$ is a complex-valued gauge field, $\Sigma$ is a 2-manifold, and $C$ is a Riemann surface endowed with a holomorphic one-form $\omega=\omega(z) d z$. It is topological along $\Sigma$ (modulo a framing anomaly), but has holomorphic dependence on $C$, and moreover has a complex gauge group, $G$. As shown in $[3,4]$, the nontrivial operators of the theory are Wilson lines, whose correlation functions realize the Yang-Baxter equation with spectral parameters, as well as the underlying Yangian algebra, quantum affine algebra, and elliptic quantum group of its rational, trigonometric and elliptic solutions, respectively. Furthermore, the boundary Yang-Baxter equation can likewise be realized by studying 4d Chern-Simons theory on an orbifold $[6,7]$.

For $C=\mathbb{R} \times S^{1}$, the $4 \mathrm{~d}$ Chern-Simons action can be dimensionally reduced along $S^{1}$ to that of $3 \mathrm{~d}$ analytically-continued Chern-Simons theory. In fact, the corresponding quantum field theories have been shown to be T-dual [8]. However, unlike ordinary 3d Chern-Simons theory, much of the work on 4d Chern-Simons relied on the path integral and Feynman diagrams alone, and no use was made of canonical quantization or holography. This was due to the infrared-free nature of $4 \mathrm{~d}$ Chern-Simons, whereby it was straightforward to deduce a local procedure to compute the expectation values of Wilson line configurations of interest. 
Nevertheless, given the importance of the 2d chiral Wess-Zumino-Witten (WZW) model dual to $3 \mathrm{~d}$ Chern-Simons theory as a straightforward example of a holographic dual, and for describing edge modes of the nonabelian fractional quantum Hall effect, it is of interest to investigate the existence of a holographic dual of 4d Chern-Simons theory. In this work, we shall indeed derive such a dual boundary theory for $4 \mathrm{~d}$ Chern-Simons on $D \times C$ (where $D$ is the disk), which turns out to be a three-dimensional analogue of the 2d chiral WZW model.

We shall focus on the boundary dual of $4 \mathrm{~d}$ Chern-Simons with $C=\mathbb{C}$, which is known to give rise to rational solutions of the Yang-Baxter equation [3]. These $R$-matrices are intertwining operators for representations of the Yangian algebra, and thus the classical integrable lattice models of concern are equivalent to Heisenberg XXX quantum spin chains. For example, one such lattice model is the rational six-vertex model, which is equivalent to the $\mathrm{XXX}_{1 / 2}$ spin chain. As we shall see, the $3 \mathrm{~d}$ "chiral" WZW model we derive furnishes an alternative and convenient method for computing the rational $R$-matrices explicitly.

Given a $3 \mathrm{~d}$ analogue of the $2 \mathrm{~d}$ chiral WZW model, the first natural question to ask is if it admits a current algebra analogous to an affine Kac-Moody algebra. In section 2, we shall show that this is indeed the case, i.e., the 3d "chiral" WZW model furnishes a particular limit of an "analytically-continued" toroidal Lie algebra.

One would also like to verify that the boundary theory captures the correlation functions of the bulk theory. For instance, the bulk correlator of two straight, perpendicular Wilson lines along $\Sigma$, at points $z_{1}$ and $z_{2}$ on $\mathbb{C}$, and in representations $R_{1}$ and $R_{2}$ of the generators of $G$, realizes the $R$-matrix, i.e., it is computed to be

$$
\widetilde{R}_{12}\left(z_{1}-z_{2}\right)=\mathbb{1}+\frac{\hbar}{z_{1}-z_{2}} T_{R_{1}}^{a} \otimes T_{R_{2} a}+O\left(\hbar^{2}\right)
$$

to linear order in $\hbar$, with the full expression for the $R$-matrix following from general theorems $[9,10]$. It would be satisfying to obtain this result solely from the boundary theory, and indeed, this is what we do in section 3 by evaluating a four-point function of local boundary operators. Furthermore, via the boundary theory, we demonstrate the topological invariance along $D$ of the bulk correlator at order $\hbar$.

We also explain how one may explicitly derive higher order contributions to the $R$ matrix, and demonstrate this by computing the $\hbar^{2}$ contribution to the correlation function of two straight, perpendicular Wilson lines explicitly. Subsequently, we show that the boundary theory also reproduces the order $\hbar$ correlation function of a pair of parallel Wilson lines. Finally, we consider three Wilson lines, all crossing each other, and show, modulo the framing anomaly, that their order $\hbar^{2}$ correlation function is reproduced by a six-point function in the boundary theory.

\section{3d "Chiral" WZW model}

Let us start with $4 \mathrm{~d}$ Chern-Simons theory with complex gauge group, $G$, defined on $\Sigma \times C$, where $\Sigma$ is a disk, denoted $D$, and $C$ is the complex plane, $\mathbb{C}$. Its action is

$$
S=\frac{1}{2 \pi \hbar} \int_{D \times \mathbb{C}} d z \wedge \operatorname{Tr}\left(\mathcal{A} \wedge d \mathcal{A}+\frac{2}{3} \mathcal{A} \wedge \mathcal{A} \wedge \mathcal{A}\right)
$$


Here, $\mathcal{A}$ can be understood to be the partial connection

$$
\mathcal{A}=\mathcal{A}_{r} d r+\mathcal{A}_{\varphi} d \varphi+\mathcal{A}_{\bar{z}} d \bar{z},
$$

where $(r, \varphi)$ are polar coordinates on $D$ and $(z, \bar{z})$ are complex coordinates on $\mathbb{C}$.

Let us first vary the action to find the equations of motion. Doing so, one finds

$$
\delta S=\frac{1}{2 \pi \hbar} \int_{D \times \mathbb{C}} d z \wedge \operatorname{Tr}(\delta \mathcal{A} \wedge \mathcal{F}+d(\delta \mathcal{A} \wedge \mathcal{A})) .
$$

The second term of the variation is a boundary term via Stoke's theorem. In order to ensure that we have equations of motion free from boundary corrections, we shall impose the boundary condition $\mathcal{A}_{\bar{z}}=0$, whereupon the boundary term vanishes.

This boundary condition is also necessary to achieve gauge invariance in the presence of boundaries. It can be shown that (2.1) is equivalent to

$$
S=-\frac{1}{2 \pi \hbar} \int_{D \times \mathbb{C}} z \operatorname{Tr}(F \wedge F)+\frac{1}{2 \pi \hbar} \int_{\partial D \times \mathbb{C}} z \operatorname{Tr}\left(\mathcal{A} \wedge d \mathcal{A}+\frac{2}{3} \mathcal{A} \wedge \mathcal{A} \wedge \mathcal{A}\right),
$$

where $\mathcal{A}$ has been extended to a full connection over $D \times \mathbb{C}$, i.e., $\mathcal{A}=\mathcal{A}_{r} d r+\mathcal{A}_{\varphi} d \varphi+$ $\mathcal{A}_{z} d z+\mathcal{A}_{\bar{z}} d \bar{z}$. The boundary term on the r.h.s. of (2.4) depends only on the components $\mathcal{A}_{\varphi}, \mathcal{A}_{z}$ and $\mathcal{A}_{\bar{z}}$, and vanishes using the boundary conditions $\mathcal{A}_{\bar{z}}=0$ and $\mathcal{A}_{z}=0$. The remaining term is gauge invariant under large gauge transformations, i.e.,

$$
\mathcal{A} \rightarrow U \mathcal{A} U^{-1}-d U U^{-1} .
$$

However, we ought to restrict $U$ such that the boundary conditions $\mathcal{A}_{\bar{z}}=\mathcal{A}_{z}=0$ are preserved. We shall achieve this by insisting that $U$ tends to the identity element of $G$ at the boundary.

Now, having imposed the boundary condition $\mathcal{A}_{\bar{z}}=0$, the action (2.1) is equivalent to

$$
\frac{1}{2 \pi \hbar} \int d z \wedge d r \wedge d \varphi \wedge d \bar{z} \operatorname{Tr}\left(2 \mathcal{A}_{\bar{z}} \mathcal{F}_{r \varphi}-\mathcal{A}_{r} \partial_{\bar{z}} \mathcal{A}_{\varphi}+\mathcal{A}_{\varphi} \partial_{\bar{z}} \mathcal{A}_{r}\right),
$$

upon integration by parts. Varying the Lagrange multiplier field, $\mathcal{A}_{\bar{z}}$, implements the constraint $\mathcal{F}_{r \varphi}=0$, which is solved by

$$
\mathcal{A}_{r}=-\partial_{r} g g^{-1}, \quad \mathcal{A}_{\varphi}=-\partial_{\varphi} g g^{-1},
$$

where $g$ is a map $g: D \times \mathbb{C} \rightarrow G$.

Changing variables from $\mathcal{A}_{r}$ and $\mathcal{A}_{\varphi}$ to $g$ in the functional integral, we note that, just as in 3d Chern-Simons theory [11], no Jacobian appears when transforming the measure, i.e.,

$$
\frac{1}{\operatorname{vol} G} \int D \mathcal{A}_{r} D \mathcal{A}_{\varphi} \delta\left(\mathcal{F}_{r \varphi}\right)=\frac{1}{\operatorname{vol} G} \int D g
$$

where the expression on the r.h.s. is the relevant Haar measure, divided by the volume of the gauge group. Furthermore, substituting the solutions (2.7) into (2.6), we obtain the action

$S(g)=\frac{1}{2 \pi \hbar} \int_{S^{1} \times \mathbb{C}} d \varphi \wedge d z \wedge d \bar{z} \operatorname{Tr}\left(\partial_{\varphi} g g^{-1} \partial_{\bar{z}} g g^{-1}\right)+\frac{1}{6 \pi \hbar} \int_{D \times \mathbb{C}} d z \wedge \operatorname{Tr}\left(d g g^{-1} \wedge d g g^{-1} \wedge d g g^{-1}\right)$, 
which takes the form of a three-dimensional analogue of the $2 \mathrm{~d}$ chiral WZW model. Now, a large gauge transformation (2.5) amounts to $g \rightarrow U g$ in (2.7). As a result, we may change the value of $g$ in the interior without changing its value at the boundary, so (2.9) only depends on $g$ at the boundary. Note that in the 2d WZW model dual to 3d Chern-Simons theory on $D \times \mathbb{R}$ with a compact gauge group studied in [11], the fact that the WessZumino term does not depend on the choice of extension of $g$ over a 3-manifold requires quantization of the WZW level/Chern-Simons coupling. However, the coupling $\hbar$ that appears in (2.9) is not quantized. This difference stems from the fact that invariance of the 3d Chern-Simons action under large gauge transformations of the form (2.5) (which leads to the Wess-Zumino term being independent of the choice of extension) requires such a quantization, while large gauge invariance of the $4 \mathrm{~d}$ Chern-Simons action does not require such a quantization, as shown below (2.4). The fact that (2.9) only depends on $g$ at the boundary implies that we can divide out the volume of the gauge group to obtain the path integral

$$
\int D g e^{i S(g)}
$$

where $g$ is now the map $g: \partial D \times \mathbb{C} \rightarrow G$.

Varying the action (2.9) gives us

$$
\delta S=-\frac{1}{\pi \hbar} \int d \varphi \wedge d z \wedge d \bar{z} \operatorname{Tr}\left(g^{-1} \delta g \partial_{\varphi}\left(g^{-1} \partial_{\bar{z}} g\right)\right),
$$

whereby we obtain the classical equation of motion

$$
\partial_{\varphi}\left(g^{-1} \partial_{\bar{z}} g\right)=0
$$

which is equivalent to $\partial_{\bar{z}}\left(\partial_{\varphi} g g^{-1}\right)=0$, and is solved by

$$
g(z, \bar{z}, \varphi)=A(z, \varphi) B(z, \bar{z}) .
$$

The equations $\partial_{\varphi}\left(g^{-1} \partial_{\bar{z}} g\right)=0$ and $\partial_{\bar{z}}\left(\partial_{\varphi} g g^{-1}\right)=0$ are in fact equivalent to the current conservation equations for the symmetry of the action under the transformation

$$
g(\varphi, z, \bar{z}) \rightarrow \tilde{\Omega}(\varphi, z) g \Omega(z, \bar{z})
$$

where $\tilde{\Omega}$ and $\Omega$ give rise to the conserved currents $J_{\varphi}=-\frac{1}{\pi \hbar} \partial_{\varphi} g g^{-1}$ and $J_{\bar{z}}=-\frac{1}{\pi \hbar} g^{-1} \partial_{\bar{z}} g$, respectively.

\subsection{Current algebra via canonical quantization}

We are now interested in computing a Poisson bracket involving the expression $J_{\varphi}=$ $-\frac{1}{\pi \hbar} \partial_{\varphi} g g^{-1}$, which we shall eventually use to obtain a quantum mechanical commutation relation in the form of a current algebra. In what follows, we shall take $\bar{z}$ to be the (complex) time direction.

Now, given an arbitrary action that is first order in the time derivative with dynamical variables $\phi^{i}$, i.e.,

$$
I=\int d t \mathscr{A}(\phi) \frac{d \phi^{i}}{d t},
$$


its variation takes the form

$$
\delta I=\int d t \omega_{i j} \delta \phi^{i} \frac{d \phi^{j}}{d t}
$$

where $\omega_{i j}=\frac{\partial}{\partial \phi^{i}} \mathscr{A}_{j}-\frac{\partial}{\partial \phi^{j}} \mathscr{A}_{i}$ is the symplectic structure on the classical phase space. The Poisson bracket of any two functions $X$ and $Y$ on the phase space is then defined by

$$
[X, Y]_{P B}=\omega^{i j} \frac{\partial X}{\partial \phi^{i}} \frac{\partial Y}{\partial \phi^{j}},
$$

where $\omega^{j k} \omega_{k l}=\delta_{l}^{j}[12]$.

For the 3d "chiral" WZW model, the variation (2.11) implies that its phase space symplectic structure is given by $\omega=1_{\mathfrak{g}} \otimes \frac{(-1)}{\pi \hbar} \frac{\partial}{\partial \varphi} \otimes 1_{z}$, where $1_{\mathfrak{g}}$ acts on the Lie algebra index, $\frac{(-1)}{\pi \hbar} \frac{\partial}{\partial \varphi}$ acts on the $\varphi$ coordinate, and $1_{z}$ acts on the $z$ coordinate. Its inverse is therefore

$$
\omega^{-1}=1_{\mathfrak{g}} \otimes(-\pi \hbar)\left(\frac{\partial}{\partial \varphi}\right)^{-1} \otimes 1_{z}
$$

Let us now compute the Poisson bracket of $X=\operatorname{Tr} A \frac{\partial g}{\partial \varphi} g^{-1}(\varphi, z)$ and $Y=\operatorname{Tr} B \frac{\partial g}{\partial \varphi^{\prime}} g^{-1}\left(\varphi^{\prime}, z^{\prime}\right)$, where $A$ and $B$ are arbitrary generators of the group $G$. In the notation of (2.15), this can be done by evaluating $\delta X \delta Y=\frac{\partial X}{\partial \phi^{i}} \frac{\partial Y}{\partial \phi^{j}} \delta \phi^{i} \delta \phi^{j}$, and subsequently replacing $\delta \phi^{i} \delta \phi^{j}$ by $\omega^{i j}$. Proceeding in this manner, we find

$$
\delta X \delta Y=\operatorname{Tr} g^{-1}(\varphi, z) A g(\varphi, z) \frac{\partial}{\partial \varphi}\left(g^{-1} \delta g(\varphi, z)\right) \cdot \operatorname{Tr} g^{-1}\left(\varphi^{\prime}, z^{\prime}\right) B g\left(\varphi^{\prime}, z^{\prime}\right) \frac{\partial}{\partial \varphi^{\prime}}\left(g^{-1} \delta g\left(\varphi^{\prime}, z^{\prime}\right)\right) .
$$

To obtain the Poisson bracket, we ought to replace $\left(g^{-1} \delta g(\varphi, z)\right)^{a}\left(g^{-1} \delta g\left(\varphi^{\prime}, z^{\prime}\right)\right)^{b}$ (where $a$ and $b$ are Lie algebra indices) by

$$
\delta^{a b}(-\pi \hbar) \theta\left(\varphi-\varphi^{\prime}\right) \delta\left(z-z^{\prime}\right)
$$

where $\theta\left(\varphi-\varphi^{\prime}\right)$ is an inverse of $\frac{\partial}{\partial \varphi}$.

Therefore, $\frac{\partial}{\partial \varphi}\left(g^{-1} \delta g(\varphi, z)\right)^{a} \cdot \frac{\partial}{\partial \varphi^{\prime}}\left(g^{-1} \delta g\left(\varphi^{\prime}, z^{\prime}\right)\right)^{b}$ in (2.19) ought to be replaced by $\delta^{a b} \pi \hbar \delta^{\prime}\left(\varphi-\varphi^{\prime}\right) \delta\left(z-z^{\prime}\right)$. Hence, we arrive at the Poisson bracket

$$
\begin{aligned}
{[X, Y]_{P B} } & =\pi \hbar \delta^{\prime}\left(\varphi-\varphi^{\prime}\right) \delta\left(z-z^{\prime}\right) \operatorname{Tr} g^{-1}(\varphi, z) A g(\varphi, z) g^{-1}\left(\varphi^{\prime}, z^{\prime}\right) B g\left(\varphi^{\prime}, z^{\prime}\right) \\
& =\pi \hbar \delta\left(\varphi-\varphi^{\prime}\right) \delta\left(z-z^{\prime}\right) \operatorname{Tr}\left([A, B] \frac{\partial g}{\partial \varphi} g^{-1}\right)+\pi \hbar \delta^{\prime}\left(\varphi-\varphi^{\prime}\right) \delta\left(z-z^{\prime}\right) \operatorname{Tr} A B .
\end{aligned}
$$

Upon rescaling both sides of this equation by $\left(\frac{1}{-\pi \hbar}\right)^{2}$, we arrive at a classical current algebra. Note that we have found a central extension term at the classical level. This is analogous to the case of the standard 2d WZW model with kinetic term in "chiral" form, i.e., using lightcone coordinates, wherein such a central extension term also appears in the classical Poisson bracket involving currents when taking one of the lightcone directions to be time, as shown in [12]. 
In the quantum theory, the Poisson bracket (2.21) corresponds to a canonical commutation relation that takes the form

$$
[X, Y]=-i \pi \hbar \delta\left(\varphi-\varphi^{\prime}\right) \delta\left(z-z^{\prime}\right) \operatorname{Tr}\left([A, B] \frac{\partial g}{\partial \varphi} g^{-1}\right)-i \pi \hbar \delta^{\prime}\left(\varphi-\varphi^{\prime}\right) \delta\left(z-z^{\prime}\right) \operatorname{Tr} A B+\ldots
$$

Note that $\hbar$ here is a coupling, based on the definition of the action in (2.1), and we have replaced the Poisson bracket $[X, Y]_{P B}$ by $i[X, Y]$. If we instead set $\hbar$ to 1 in the action and replace the Poisson bracket $[X, Y]_{P B}$ by $\underset{\widetilde{\hbar}}{\stackrel{i}{[}}[X, Y]$, where $\widetilde{\hbar}$ is Planck's constant, then we will obtain $(2.22)$ with $\hbar$ replaced by $\widetilde{\hbar}$. The terms represented by the ellipsis in $(2.22)$ indicate possible higher order terms in Planck's constant.

As we shall explain now, such quantum corrections to the classical current algebra are expected to be fixed by a symmetry of the quantum 3d WZW model. Firstly, for $C=\mathbb{C}$, the bulk $4 \mathrm{~d}$ Chern-Simons theory computes the rational R-matrix, that has $G$ as an automorphism group, and therefore its dual quantum 3d WZW model ought to have $G$ as a symmetry. As we have seen in (2.14), there are two $G$ transformations that leave (2.9) invariant, but the one defined by right multiplication of $g$ by $\Omega$ is in fact a redundancy arising from the form of the parametrization of $A$ by $g$ in (2.7), and ought to be fixed. The relevant $G$ symmetry therefore ought to arise from left multiplication of $g$ by $\widetilde{\Omega}$, with conserved current $J_{\varphi}$, and this should be preserved in the quantum theory.

In fact, rewriting (2.22) as

$$
\begin{aligned}
{\left[\operatorname{Tr} A J_{\varphi}(\varphi, z), \operatorname{Tr} B J_{\varphi}\left(\varphi^{\prime}, z^{\prime}\right)\right]=} & i \delta\left(\varphi-\varphi^{\prime}\right) \delta\left(z-z^{\prime}\right) \operatorname{Tr}[A, B] J_{\varphi}(\varphi, z) \\
& -\frac{i}{\pi \hbar} \delta^{\prime}\left(\varphi-\varphi^{\prime}\right) \delta\left(z-z^{\prime}\right) \operatorname{Tr} A B+\ldots
\end{aligned}
$$

and integrating over $\varphi, \varphi^{\prime}, z$ and $z^{\prime}$ on both sides, we find that $Q=\int d \varphi \int d z J_{\varphi}(\varphi, z)$ generates this quantum $G$ symmetry if the quantum corrections are restricted to be total derivatives, i.e., it satisfies

$$
\left[Q^{a}, Q^{b}\right]=i f_{c}^{a b} Q^{c}
$$

Moreover, since $Q$ generates this symmetry,

$$
\left[J_{\varphi}^{a}(\varphi, z), g\left(\varphi^{\prime}, z^{\prime}\right)\right]=-i T^{a} g\left(\varphi^{\prime}, z^{\prime}\right) \delta\left(\varphi-\varphi^{\prime}\right) \delta\left(z-z^{\prime}\right)
$$

ought to hold up to total derivatives, since this would imply $i \widetilde{\omega}_{a}\left[Q^{a}, g\left(\varphi^{\prime} z^{\prime}\right)\right]=\widetilde{\omega}_{a} T^{a} g\left(\varphi^{\prime}, z^{\prime}\right)$, which is the infinitesimal form of the left multiplication of $g$. Note that since the corrections to (2.23) and (2.25) are restricted to be total derivatives (or repeated total derivatives) by the $G$ symmetry, these corrections must be multiplied by positive powers of $\hbar$ (which has dimensions of length) to satisfy dimensional analysis. We expect that the explicit form of these corrections, that are higher order in $\hbar$, can be found via a Moyal quantization scheme that preserves the $G$ symmetry.

To express (2.23) in a more familiar form, we expand the currents in terms of their Fourier modes along $S^{1}$,

$$
J_{\varphi}(\varphi, z)=\frac{1}{2 \pi} \sum_{n=-\infty}^{\infty} J_{\varphi}^{n}(z) e^{i n \varphi}
$$


and utilize the orthogonality of these modes, which leads us to

$$
\left[\operatorname{Tr} A J_{\varphi}^{n}(z), \operatorname{Tr} B J_{\varphi}^{m}\left(z^{\prime}\right)\right]=i \operatorname{Tr}[A, B] J_{\varphi}^{n+m}(z) \delta\left(z-z^{\prime}\right)+\frac{2}{\hbar}\left(n \delta_{m+n, 0}\right) \delta\left(z-z^{\prime}\right) \operatorname{Tr} A B+\ldots
$$

This algebra has the form of a Kac-Moody algebra with generators having holomorphic dependence on the Riemann surface, $\mathbb{C}$. Note that there is no quantization condition on $\hbar$ here, unlike the current algebra derived from the boundary theory of ordinary $3 \mathrm{~d}$ Chern-Simons theory.

To further understand this algebra, we shall write $z=\epsilon t+i \theta$, compactify the $\theta$ direction to be valued in $[0,2 \pi]$, and subsequently take $\epsilon \rightarrow 0$. Upon doing so, we may perform another expansion in Fourier modes, i.e.,

$$
J_{\varphi}^{n}(\theta)=\frac{1}{2 \pi} \sum_{\tilde{n}=-\infty}^{\infty} J_{\varphi}^{n, \tilde{n}} e^{i \tilde{n} \theta} .
$$

Then, employing the orthogonality of these modes, the resulting algebra takes the form

$$
\left[\operatorname{Tr} A J_{\varphi}^{n, \tilde{n}}, \operatorname{Tr} B J_{\varphi}^{m, \tilde{m}}\right]=i \operatorname{Tr}[A, B] J_{\varphi}^{n+m, \tilde{n}+\tilde{m}}+\frac{4 \pi}{\hbar} n \delta_{m+n, 0} \delta_{\tilde{m}+\tilde{n}, 0} \operatorname{Tr} A B+\ldots
$$

This has the form of a two-toroidal Lie algebra (or a centrally-extended double loop algebra), which, in particular, arises as the current algebra of the four-dimensional WZW model studied in [13-16]. Hence, the algebra (2.27) that we obtained can be understood to be an 'analytical continuation' of the two-toroidal Lie algebra (2.29), with one of the two 'loop directions' decompactified. This is not surprising, considering the fact that $4 \mathrm{~d}$ Chern-Simons theory for $C=\mathbb{R} \times S^{1}$ can be understood to be 3d Chern-Simons theory for the loop group, but with the 'loop direction' complexified [17].

A further comparison with the four-dimensional WZW model is in order. Firstly, note that $4 \mathrm{~d}$ Chern-Simons theory is unrenormalizable by power-counting, and so is its dual 3d "chiral" WZW model, since in both cases the loop-counting parameter, $\hbar$, has dimensions of inverse mass. Nevertheless, it has been shown that $4 \mathrm{~d}$ Chern-Simons theory can be quantized in perturbation theory using BV quantization [2], ensuring that it is finite. Now, the 4d WZW model is one-loop finite (and is likely to be all-loop finite) despite being unrenormalizable by power-counting [18], and it also admits an infinite-dimensional current algebra. The presence of this algebra appears to be responsible for the one-loop finiteness, since this is the only four-dimensional nonlinear sigma model that is one-loop finite apart from the sigma model on $S^{7}$. Thus, the infinite-dimensional algebra (2.27) should likewise give rise to the finiteness of the $3 \mathrm{~d}$ "chiral" WZW model, and therefore of $4 \mathrm{~d}$ Chern-Simons theory. It would be of interest to explore this further.

Finally, we shall also compare our 3d WZW model with the 2d integrable sigma models of Delduc, Lacroix, Magro and Vicedo [19] that admit descriptions as affine Gaudin models, and are obtained from 4d Chern-Simons theory via the approach of Costello and Yamazaki [5]. As an intermediate step towards deriving these sigma models in [19], the action

$$
S=\frac{i}{12 \pi} \int_{\Sigma \times \mathbb{C} P^{1}} \omega \wedge\left\langle\widehat{g}^{-1} d \widehat{g}, \widehat{g}^{-1} d \widehat{g} \wedge \widehat{g}^{-1} d \widehat{g}\right\rangle+\frac{i}{4 \pi} \int_{\Sigma \times \mathbb{C} P^{1}} d \omega \wedge\left\langle\widehat{g}^{-1} d \widehat{g}, \mathcal{L}\right\rangle,
$$


was obtained from $4 \mathrm{~d}$ Chern-Simons theory (where we use the notation and conventions of [19]) via $A=-d \widehat{g} \widehat{g}^{-1}+\widehat{g} \mathcal{L} \widehat{g}^{-1}$, where $\mathcal{L}$ is interpreted as a Lax connection. Now, if we include a boundary for $\Sigma$ by setting $\Sigma=D$, one finds the additional term

$$
-\frac{i}{4 \pi} \int_{\partial \Sigma \times \mathbb{C} P^{1}} \omega \wedge\left\langle\widehat{g}^{-1} d \widehat{g}, \mathcal{L}\right\rangle
$$

via this procedure. To relate to our $3 \mathrm{~d}$ WZW model, we shall set $\omega=d z$, and we shall pick $\mathcal{L}=-\partial_{\varphi} \widetilde{g} \widetilde{g}^{-1} d \varphi$ for a map $\widetilde{g} \rightarrow \partial \Sigma \times \mathbb{C} P^{1}$, where $\mathcal{L}_{\varphi}$ obeys $\partial_{r} \mathcal{L}_{\varphi}=0$ and $\partial_{\bar{z}} \mathcal{L}_{\varphi}=0$ (which is reminiscent of the current conservation equation for $J_{\varphi}$ ). The full action can be written as

$$
S=\frac{i}{12 \pi} \int_{\Sigma \times \mathbb{C} P^{1}} d z \wedge\left\langle\widehat{g}^{-1} d \widehat{g}, \widehat{g}^{-1} d \widehat{g} \wedge \widehat{g}^{-1} d \widehat{g}\right\rangle+\frac{i}{4 \pi} \int_{\partial \Sigma \times \mathbb{C} P^{1}} d z \wedge\left\langle\widehat{g}^{-1} d \widehat{g}, \partial_{\varphi} \widetilde{g} \widetilde{g}^{-1} d \varphi\right\rangle,
$$

where we have used the fact that $d(d z)=0$ everywhere except $z \rightarrow \infty$, which is where we take $\widetilde{g} \rightarrow \mathbb{1}$ (the same boundary condition was previously used implicitly for the field $g$ ). Then, taking $\widehat{g}=\bar{g}^{-1}$, and restricting $\bar{g}$ to be equal to $\widetilde{g}$ along $\partial \Sigma$, we find

$$
S=-\frac{i}{12 \pi} \int_{\Sigma \times \mathbb{C} P^{1}} d z \wedge\left\langle d \bar{g} \bar{g}^{-1}, d \bar{g} \bar{g}^{-1} \wedge d \bar{g} \bar{g}^{-1}\right\rangle-\frac{i}{4 \pi} \int_{\partial \Sigma \times \mathbb{C} P^{1}} d \varphi \wedge d z \wedge d \bar{z}\left\langle\partial_{\bar{z}} \widetilde{g} \widetilde{g}^{-1}, \partial_{\varphi} \widetilde{g} \widetilde{g}^{-1}\right\rangle,
$$

which is a $3 \mathrm{~d}$ "chiral" WZW action, where the equation of motion $\partial_{\bar{z}}\left(\partial_{\varphi} \widetilde{g} \widetilde{g}^{-1}\right)=0$ has been implemented. ${ }^{1}$ Thus, an analogous derivation starting with $4 \mathrm{~d}$ Chern-Simons theory on $D \times \mathbb{C} P^{1}$ with a more general choice of $\omega$ ought to lead to a generalization of the $3 \mathrm{~d}$ WZW model, which we expect to be equivalent, via a generalization of the approach of $[5,19]$, to a $2 \mathrm{~d}$ integrable sigma model with a $1 \mathrm{~d}$ boundary action. Based on the results of $[19,20]$, this integrable sigma model is likely to be described by some type of affine Gaudin model.

\section{Wilson lines and boundary local operators}

We shall now describe Wilson lines in 4d Chern-Simons theory in terms of local operators of its boundary dual. This is possible due to the flatness condition that restricts the gauge field components on $D$ to be pure gauge configurations, as shown in (2.7). To see this, note that a Wilson line along a curve $\mathcal{C}$, starting at $t_{i}$ and ending at $t_{f}$, and in a representation $R$, satisfies

$$
\mathcal{P} e^{\int_{\mathcal{C}} \mathcal{A}}=g_{R}^{-1}\left(t_{f}\right) \mathcal{P} e^{\int_{\mathcal{C}} \mathcal{A}^{\prime}} g_{R}\left(t_{i}\right)
$$

where $\mathcal{A}=g \mathcal{A}^{\prime} g^{-1}-d g g^{-1}$. Setting $\mathcal{A}^{\prime}=0$, we find that

$$
\mathcal{P} e^{\int_{\mathcal{C}}\left(-d g g^{-1}\right)}=g_{R}^{-1}\left(t_{f}\right) g_{R}\left(t_{i}\right) .
$$

If $t_{f}$ and $t_{i}$ are points on $\partial D=S^{1}$, this implies that a bulk Wilson line operator can be completely described in terms of a pair of local boundary operators. In fact, such boundaryanchored Wilson lines are automatically gauge invariant, since all gauge transformations are trivial at the boundary.

\footnotetext{
${ }^{1}$ Note that using our earlier conventions for the $4 \mathrm{~d}$ Chern-Simons action, we can obtain the 3d WZW action (2.9) in the same manner, multiplied by a minus sign that can be absorbed into the definition of $\hbar$.
} 


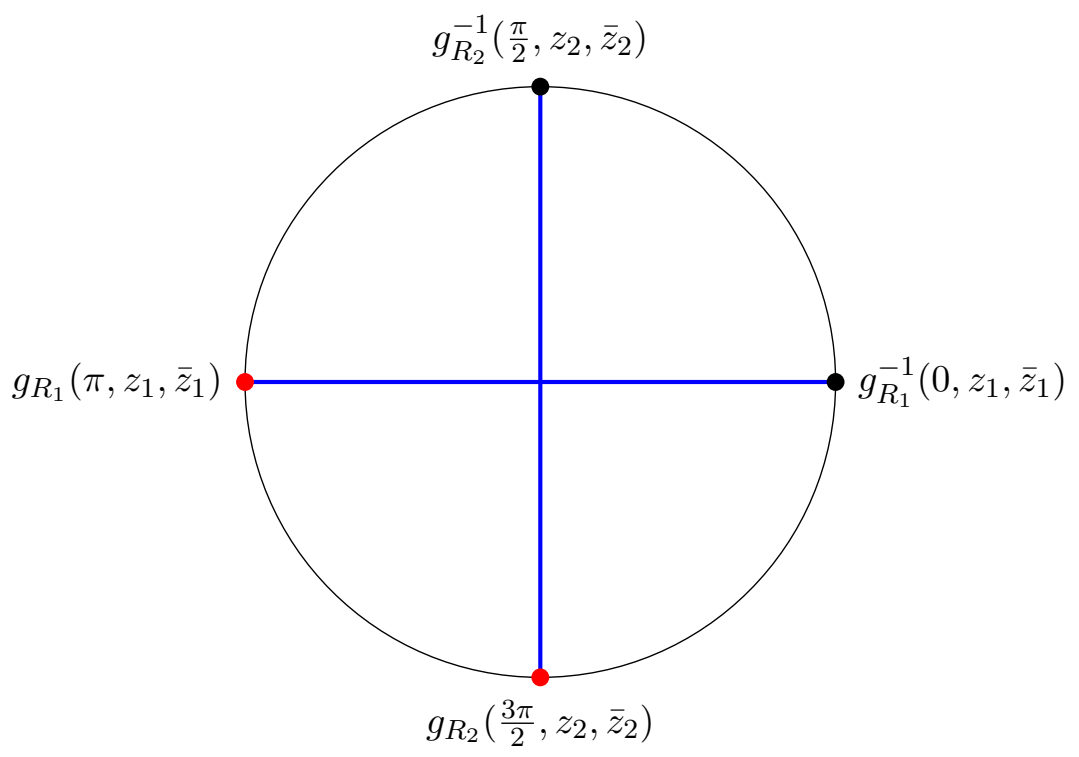

Figure 1. Perpendicular Wilson lines on $D$.

This suggests that correlation functions of Wilson line operators in $4 \mathrm{~d}$ Chern-Simons theory can be captured by correlation functions of local operators in the $3 \mathrm{~d}$ "chiral" WZW model. This includes correlators of crossed Wilson lines which compute the $R$-matrices of integrable lattice models. We shall now attempt to verify this.

In the bulk computation by Costello, Witten, and Yamazaki [3], the order $\hbar$ contribution to the $R$-matrix was found by performing perturbation theory around the trivial field configuration, $\mathcal{A}=0$, and computing free-field propagators between Wilson lines. In the same vein, we shall consider perturbation theory around the field configuration $g=\mathbb{1}$, and we shall use a free-field propagator to compute the relevant correlation function involving operators appearing on the right hand side of (3.2), i.e.,

$$
\begin{aligned}
& \left\langle\mathcal{P} e^{\int_{\varphi=\pi}^{\varphi=0} \mathcal{A}_{R_{1}}\left(z_{1}, \bar{z}_{1}\right)} \otimes \mathcal{P} e^{\int_{\varphi=3 \pi / 2}^{\varphi=\pi / 2} \mathcal{A}_{R_{2}}\left(z_{2}, \bar{z}_{2}\right)}\right\rangle \\
= & \left\langle g_{R_{1}}^{-1}\left(0, z_{1}, \bar{z}_{1}\right) g_{R_{1}}\left(\pi, z_{1}, \bar{z}_{1}\right) \otimes g_{R_{2}}^{-1}\left(\pi / 2, z_{2}, \bar{z}_{2}\right) g_{R_{2}}\left(3 \pi / 2, z_{2}, \bar{z}_{2}\right)\right\rangle .
\end{aligned}
$$

The relevant operators are depicted in figure 1.

Now, we may expand the field, $g$, as

$$
g=e^{\phi_{a} T^{a}}=\mathbb{1}+\phi_{a} T^{a}+\ldots
$$

The free part of the 3d WZW action is then

$$
\frac{1}{2 \pi \hbar} \int_{S^{1} \times \mathbb{C}} d \varphi \wedge d z \wedge d \bar{z} \operatorname{Tr}\left(\partial_{\varphi} g g^{-1} \partial_{\bar{z}} g g^{-1}\right)=-\frac{1}{2 \pi \hbar} \int_{S^{1} \times \mathbb{C}} d \varphi \wedge d z \wedge d \bar{z} \phi^{a} \partial_{\varphi} \partial_{\bar{z}} \phi_{a}+\ldots
$$

where we have performed integration by parts after expanding the field $g$. 
Next, from the free action, one may construct the generating functional

$$
\begin{aligned}
Z_{0}[J] & =\frac{\int D \phi e^{\frac{i}{2 \pi \hbar} \int_{S^{1} \times \mathbb{C}} d \varphi \wedge d z \wedge d \bar{z}\left(-\phi^{a} \partial_{\varphi} \partial_{\bar{z}} \phi_{a}+2 \pi i \hbar J_{a} \phi^{a}\right)}}{\int D \phi e^{\frac{i}{2 \pi \hbar} \int_{S^{1} \times \mathbb{C}} d \varphi \wedge d z \wedge d \bar{z}\left(-\phi^{a} \partial_{\varphi} \partial_{\bar{z}} \phi_{a}\right)}} \\
& =\exp \left(-\frac{2 \pi i \hbar}{4} \int d^{3} x \int d^{3} y J_{a}(x) \Delta^{a b}(x-y) J_{b}(y)\right)
\end{aligned}
$$

where $x=(\varphi, z, \bar{z}), y=\left(\varphi^{\prime}, z^{\prime}, \bar{z}^{\prime}\right)$, and $\Delta^{a b}$ is the propagator which obeys

$$
\partial_{\varphi} \partial_{\bar{z}} \Delta^{a b}(x)=\delta^{a b} \delta^{3}(x)
$$

and is given explicitly by

$$
\Delta^{a b}(x)=\delta^{a b} \frac{1}{2 \pi i} \frac{1}{z} \widetilde{\Delta}_{\varphi}
$$

where

$$
\widetilde{\Delta}_{\varphi}=\frac{1}{2 \pi}\left(\sum_{k=1}^{\infty} \frac{e^{i k \varphi}}{i k}+\varphi+\sum_{k=-\infty}^{-1} \frac{e^{i k \varphi}}{i k}\right),
$$

which satisfies $\partial_{\varphi} \widetilde{\Delta}_{\varphi}=\frac{1}{2 \pi}\left(\sum_{k=-\infty}^{\infty} e^{i k \varphi}\right)=\delta(\varphi)$. Note that (3.6) holds since

$$
\partial_{\bar{z}} \frac{1}{2 \pi i} \frac{1}{z}=\delta^{2}(z, \bar{z})
$$

Moreover, the propagator satisfies $\Delta^{a b}(x-y)=\Delta^{b a}(y-x)$. The two point function can be found from (3.5) to be

$$
\left\langle\phi^{a}(x) \phi^{b}(y)\right\rangle=-\pi i \hbar \Delta^{a b}(x-y) .
$$

Before we proceed, we first note that the propagator (3.7) has no dependence on the $\bar{z}$ coordinate. This can be understood from the fact that there is a gauge redundancy in the $3 \mathrm{~d}$ "chiral" WZW model, namely the invariance of the parametrizations (2.7) under transformations generated by $\Omega$ in (2.14). This redundancy can be fixed by setting $B(z, \bar{z})=\mathbb{1}$ in (2.13). As a result, the operator $g$ has no $\bar{z}$-dependence, and therefore correlation functions involving it should not have $\bar{z}$-dependence either.

Another pertinent point to note is that the propagator is a multi-valued function, as it includes the expression on the r.h.s. of (3.8) as a factor. Hence, to obtain a single-valued propagator, we ought to define it with a branch cut. We shall pick the branch cut to be from $r=0$ to $(r=R, \varphi=\pi)$, where $R$ is the radius of $D$. This effectively restricts $\varphi$ in $(3.8)$ to take values in $(-\pi, \pi)$. In this manner, we obtain a well-defined, single-valued propagator.

Now, to compute the r.h.s. of (3.3), we shall expand each operator to linear order in $\phi$

$$
\begin{aligned}
& \left\langle g_{R_{1}}^{-1}\left(0, z_{1}\right) g_{R_{1}}\left(\pi, z_{1}\right) \otimes g_{R_{2}}^{-1}\left(\pi / 2, z_{2}\right) g_{R_{2}}\left(3 \pi / 2, z_{2}\right)\right\rangle \\
= & \left\langle\left(\mathbb{1}-\phi_{a}\left(0, z_{1}\right) T_{R_{1}}^{a}\right)\left(\mathbb{1}+\phi_{b}\left(\pi, z_{1}\right) T_{R_{1}}^{b}\right) \otimes\left(\mathbb{1}-\phi_{c}\left(\pi / 2, z_{2}\right) T_{R_{2}}^{c}\right)\left(\mathbb{1}+\phi_{d}\left(3 \pi / 2, z_{2}\right) T_{R_{2}}^{d}\right)\right\rangle+\ldots
\end{aligned}
$$


We then only keep terms of quadratic or lower order in the fields, while taking selfcontractions (i.e., correlators of operators with the same value of $z$ ) to be zero via regularization. Also note that 1-point functions can be shown to be zero using (3.5). Hence, we find

$$
\begin{aligned}
\mathbb{1}+ & \left\langle\phi_{a}\left(0, z_{1}\right) \phi_{c}\left(\pi / 2, z_{2}\right)\right\rangle T_{R_{1}}^{a} \otimes T_{R_{2}}^{c}-\left\langle\phi_{a}\left(\pi, z_{1}\right) \phi_{c}\left(\pi / 2, z_{2}\right)\right\rangle T_{R_{1}}^{a} \otimes T_{R_{2}}^{c} \\
& -\left\langle\phi_{a}\left(2 \pi, z_{1}\right) \phi_{c}\left(3 \pi / 2, z_{2}\right)\right\rangle T_{R_{1}}^{a} \otimes T_{R_{2}}^{c}+\left\langle\phi_{a}\left(\pi, z_{1}\right) \phi_{c}\left(3 \pi / 2, z_{2}\right)\right\rangle T_{R_{1}}^{a} \otimes T_{R_{2}}^{c}+O\left(\hbar^{2}\right) \\
= & \mathbb{1}-\frac{\hbar}{2} \delta_{a c} \frac{1}{z_{1}-z_{2}} \widetilde{\Delta}_{-\frac{\pi}{2}} T_{R_{1}}^{a} \otimes T_{R_{2}}^{c}+\frac{\hbar}{2} \delta_{a c} \frac{1}{z_{1}-z_{2}} \widetilde{\Delta}_{\frac{\pi}{2}} T_{R_{1}}^{a} \otimes T_{R_{2}}^{c} \\
& +\frac{\hbar}{2} \delta_{a c} \frac{1}{z_{1}-z_{2}} \widetilde{\Delta}_{\frac{\pi}{2}} T_{R_{1}}^{a} \otimes T_{R_{2}}^{c}-\frac{\hbar}{2} \delta_{a c} \frac{1}{z_{1}-z_{2}} \widetilde{\Delta}_{-\frac{\pi}{2}} T_{R_{1}}^{a} \otimes T_{R_{2}}^{c}+O\left(\hbar^{2}\right) \\
= & \mathbb{1}+\frac{\hbar}{z_{1}-z_{2}}\left(\widetilde{\Delta}_{\frac{\pi}{2}}-\widetilde{\Delta}_{-\frac{\pi}{2}}\right) T_{R_{1}}^{a} \otimes T_{R_{2} a}+O\left(\hbar^{2}\right) \\
= & \mathbb{1}+\frac{\hbar}{z_{1}-z_{2}} T_{R_{1}}^{a} \otimes T_{R_{2} a}+O\left(\hbar^{2}\right) .
\end{aligned}
$$

Here, we have used the fact that

$$
\widetilde{\Delta}_{\frac{\pi}{2}}=\frac{1}{2 \pi} \frac{\pi}{2}+\frac{1}{\pi}\left(1-\frac{1}{3}+\frac{1}{5}-\frac{1}{7} \ldots\right)=\frac{1}{2},
$$

and likewise $\widetilde{\Delta}_{-\frac{\pi}{2}}=-\frac{1}{2}$. We have thus obtained, from our $3 \mathrm{~d}$ "chiral" WZW model, the exact order $\hbar$ correlation function for a pair of perpendicular Wilson lines that Costello, Witten and Yamazaki [3] computed via the bulk 4d Chern-Simons path integral.

\subsection{Non-perpendicular Wilson lines}

We can generalize the calculation above to the case of non-perpendicular Wilson lines. As an example, we shall start with two perpendicular Wilson lines, and rotate the vertical Wilson line clockwise by an angle, $\delta$, as shown in figure 2 .

The four-point function we should compute is

$$
\left\langle g_{R_{1}}^{-1}\left(0, z_{1}\right) g_{R_{1}}\left(\pi, z_{1}\right) \otimes g_{R_{2}}^{-1}\left(\pi / 2-\delta, z_{2}\right) g_{R_{2}}\left(3 \pi / 2-\delta, z_{2}\right)\right\rangle .
$$

Expanding each operator to linear order in $\phi$ as in (3.11), we find

$$
\begin{aligned}
\mathbb{1} & \left\langle\left\langle\phi_{a}\left(0, z_{1}\right) \phi_{c}\left(\pi / 2-\delta, z_{2}\right)\right\rangle T_{R_{1}}^{a} \otimes T_{R_{2}}^{c}-\left\langle\phi_{a}\left(\pi, z_{1}\right) \phi_{c}\left(\pi / 2-\delta, z_{2}\right)\right\rangle T_{R_{1}}^{a} \otimes T_{R_{2}}^{c}\right. \\
- & \left\langle\phi_{a}\left(2 \pi, z_{1}\right) \phi_{c}\left(3 \pi / 2-\delta, z_{2}\right)\right\rangle T_{R_{1}}^{a} \otimes T_{R_{2}}^{c}+\left\langle\phi_{a}\left(\pi, z_{1}\right) \phi_{c}\left(3 \pi / 2-\delta, z_{2}\right)\right\rangle T_{R_{1}}^{a} \otimes T_{R_{2}}^{c}+O\left(\hbar^{2}\right) \\
= & \mathbb{1}-\frac{\hbar}{2} \delta_{a c} \frac{1}{z_{1}-z_{2}} \widetilde{\Delta}_{-\frac{\pi}{2}+\delta} T_{R_{1}}^{a} \otimes T_{R_{2}}^{c}+\frac{\hbar}{2} \delta_{a c} \frac{1}{z_{1}-z_{2}} \widetilde{\Delta}_{\frac{\pi}{2}+\delta} T_{R_{1}}^{a} \otimes T_{R_{2}}^{c} \\
& +\frac{\hbar}{2} \delta_{a c} \frac{1}{z_{1}-z_{2}} \widetilde{\Delta}_{\frac{\pi}{2}+\delta} T_{R_{1}}^{a} \otimes T_{R_{2}}^{c}-\frac{\hbar}{2} \delta_{a c} \frac{1}{z_{1}-z_{2}} \widetilde{\Delta}_{-\frac{\pi}{2}+\delta} T_{R_{1}}^{a} \otimes T_{R_{2}}^{c}+O\left(\hbar^{2}\right) \\
= & \mathbb{1}+\frac{\hbar}{z_{1}-z_{2}}\left(\widetilde{\Delta}_{\frac{\pi}{2}+\delta}-\widetilde{\Delta}_{-\frac{\pi}{2}+\delta}\right) T_{R_{1}}^{a} \otimes T_{R_{2} a}+O\left(\hbar^{2}\right)
\end{aligned}
$$

Now, note that (3.8) can be rewritten as

$$
\widetilde{\Delta}_{\varphi}=\frac{\varphi}{2 \pi}+\frac{1}{\pi} \sum_{k=1}^{\infty} \frac{\sin (k \varphi)}{k} .
$$




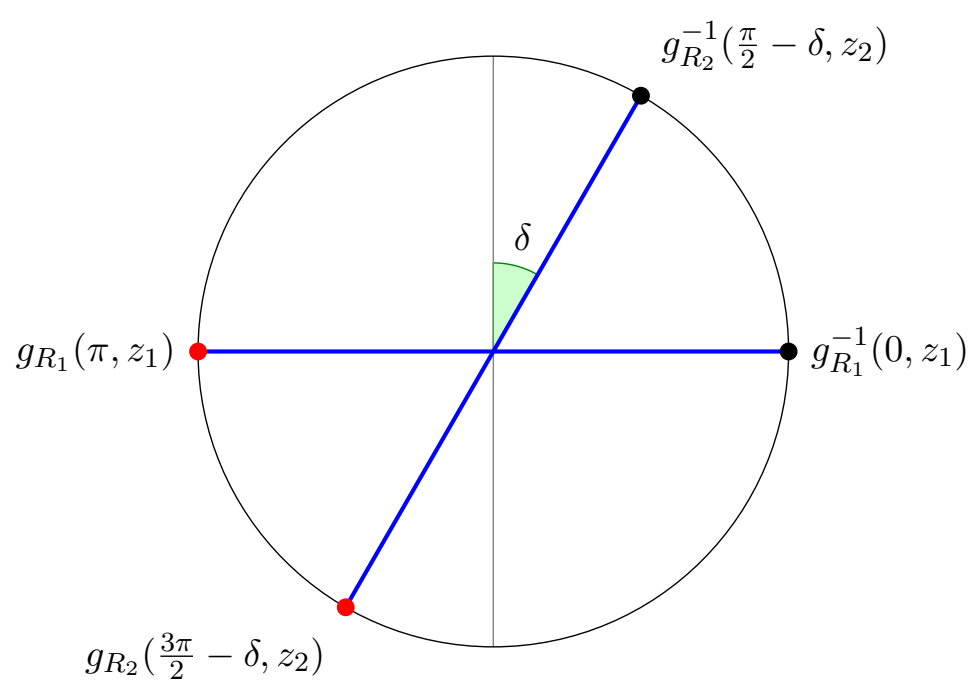

Figure 2. Non-perpendicular Wilson lines on $D$.

This implies that

$$
\widetilde{\Delta}_{\frac{\pi}{2}+\delta}=\frac{\frac{\pi}{2}+\delta}{2 \pi}+\frac{1}{\pi} \sum_{k=1}^{\infty} \frac{\sin \left(k \frac{\pi}{2}\right) \cos (k \delta)+\cos \left(k \frac{\pi}{2}\right) \sin (k \delta)}{k},
$$

and

$$
\widetilde{\Delta}_{-\frac{\pi}{2}+\delta}=\frac{-\frac{\pi}{2}+\delta}{2 \pi}-\frac{1}{\pi} \sum_{k=1}^{\infty} \frac{\sin \left(k \frac{\pi}{2}\right) \cos (k \delta)-\cos \left(k \frac{\pi}{2}\right) \sin (k \delta)}{k} .
$$

We find that the sums over $k$ above can be separated into two types of sums, each having the form of a Fourier series, namely, the Fourier series for a square wave,

$$
\sum_{k=1}^{\infty} \frac{\sin \left(\frac{k \pi}{2}\right) \cos (k x)}{k}=\frac{\pi}{4} \operatorname{sign}(\cos (x))
$$

and the Fourier series for a sawtooth wave,

$$
\sum_{k=1}^{\infty} \frac{\cos \left(\frac{k \pi}{2}\right) \sin (k x)}{k}=\frac{-x}{2}+\frac{l \pi}{2}, \quad \pi\left(l-\frac{1}{2}\right)<x<\pi\left(l+\frac{1}{2}\right), l \in \mathbb{Z},
$$

for $x \in \mathbb{R}$. However, single-valuedness of the propagators involved in the computation (3.15) requires that $-\frac{\pi}{2}<\delta<\frac{\pi}{2}$, implying

$$
\sum_{k=1}^{\infty} \frac{\sin \left(\frac{k \pi}{2}\right) \cos (k \delta)}{k}=\frac{\pi}{4}
$$

and

$$
\sum_{k=1}^{\infty} \frac{\cos \left(\frac{k \pi}{2}\right) \sin (k \delta)}{k}=-\frac{\delta}{2}
$$




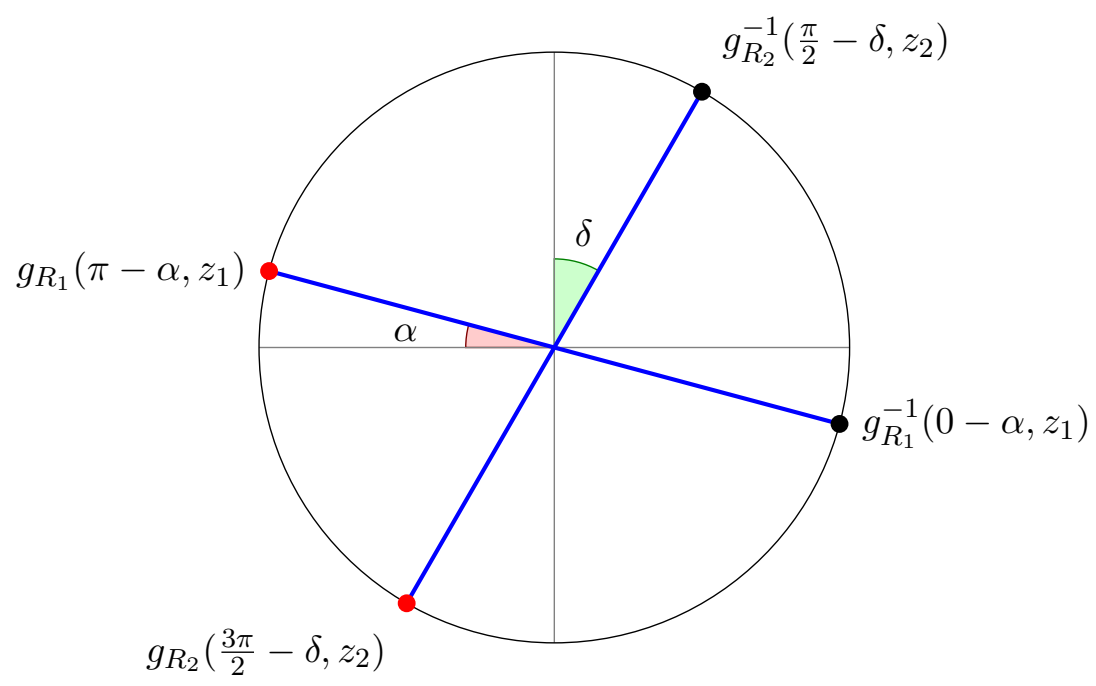

Figure 3. Crossed Wilson lines, both rotated from perpendicularity.

From here we find that

$$
\begin{aligned}
\widetilde{\Delta}_{\frac{\pi}{2}+\delta} & =\frac{1}{2} \\
\widetilde{\Delta}_{-\frac{\pi}{2}+\delta} & =-\frac{1}{2}
\end{aligned}
$$

for $-\frac{\pi}{2}<\delta<\frac{\pi}{2}$. As a result, (3.15) is in fact independent of the angle $\delta$, and agrees precisely with the result we found for perpendicular Wilson lines. Hence, we once again find agreement with the results of Costello, Witten and Yamazaki [3].

Arbitrarily crossed Wilson line. We can generalize the preceding calculations further to more general configurations of crossed Wilson lines, for which we expect to obtain the same result as (3.12) due to the topological invariance of $4 \mathrm{~d}$ Chern-Simons along $D$. For instance, we can consider both Wilson lines rotated from perpendicularity, as shown in figure 3. The corresponding four-point function is unaffected by the additional rotation, i.e., we find

$$
\begin{aligned}
& \left\langle g_{R_{1}}^{-1}\left(0-\alpha, z_{1}\right) g_{R_{1}}\left(\pi-\alpha, z_{1}\right) \otimes g_{R_{2}}^{-1}\left(\pi / 2-\delta, z_{2}\right) g_{R_{2}}\left(3 \pi / 2-\delta, z_{2}\right)\right\rangle \\
= & \mathbb{1}+\frac{\hbar}{z_{1}-z_{2}}\left(\widetilde{\Delta}_{\frac{\pi}{2}-\alpha+\delta}-\widetilde{\Delta}_{-\frac{\pi}{2}-\alpha+\delta}\right) T_{R_{1}}^{a} \otimes T_{R_{2} a}+O\left(\hbar^{2}\right) \\
= & \mathbb{1}+\frac{\hbar}{z_{1}-z_{2}} T_{R_{1}}^{a} \otimes T_{R_{2} a}+O\left(\hbar^{2}\right),
\end{aligned}
$$

(where $-\frac{\pi}{2}<-\alpha+\delta<\frac{\pi}{2}$ to ensure single-valued propagators) with the use of the identity (3.23).

A different generalization is that of perpendicular Wilson lines crossing at a point that is not the origin, $r=0$, as shown in figure 4 . The four-point function in this case is also 


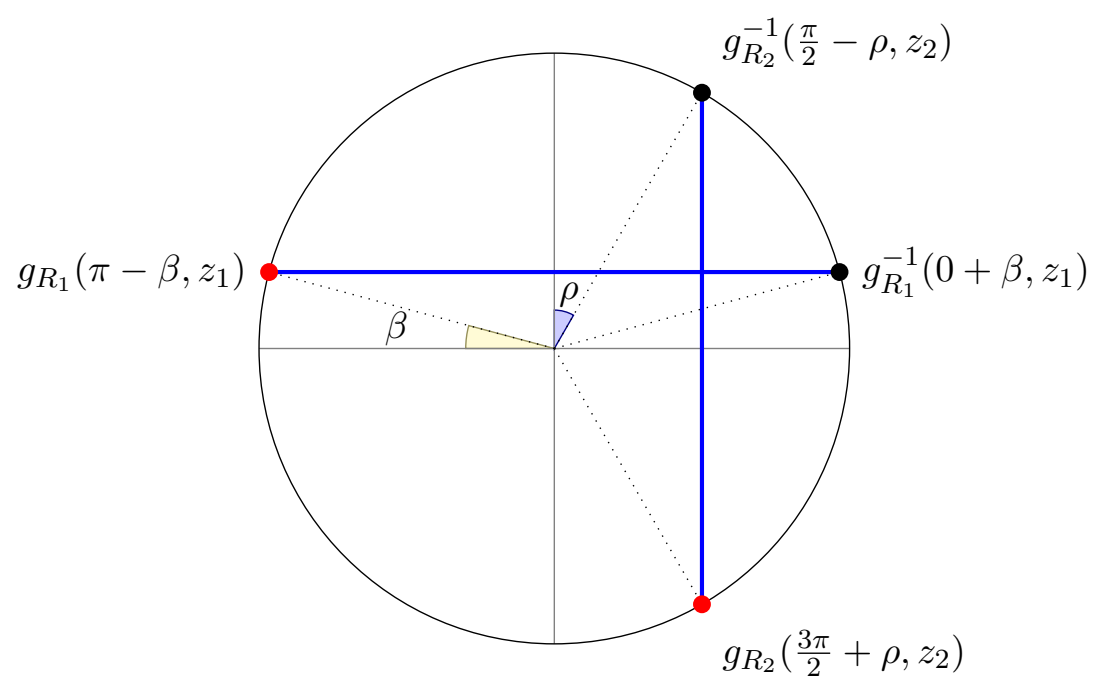

Figure 4. Perpendicular Wilson lines crossed away from the origin.

independent of the angles $\beta$ and $\rho$ shown in the figure, i.e., we have

$$
\begin{aligned}
& \left\langle g_{R_{1}}^{-1}\left(0+\beta, z_{1}\right) g_{R_{1}}\left(\pi-\beta, z_{1}\right) \otimes g_{R_{2}}^{-1}\left(\pi / 2-\rho, z_{2}\right) g_{R_{2}}\left(3 \pi / 2+\rho, z_{2}\right)\right\rangle \\
= & \mathbb{1}+\frac{\hbar}{z_{1}-z_{2}} \frac{1}{2}\left(\widetilde{\Delta}_{\frac{\pi}{2}+\beta-\rho}-\widetilde{\Delta}_{-\frac{\pi}{2}+\beta+\rho}+\widetilde{\Delta}_{\frac{\pi}{2}-\beta+\rho}-\widetilde{\Delta}_{-\frac{\pi}{2}-\beta-\rho}\right) T_{R_{1}}^{a} \otimes T_{R_{2} a}+O\left(\hbar^{2}\right) \\
= & \mathbb{1}+\frac{\hbar}{z_{1}-z_{2}} T_{R_{1}}^{a} \otimes T_{R_{2} a}+O\left(\hbar^{2}\right),
\end{aligned}
$$

using the identity (3.23), where $-\frac{\pi}{2}<\beta+\rho<\frac{\pi}{2}$ and $-\frac{\pi}{2}<\beta-\rho<\frac{\pi}{2}$ to ensure singlevaluedness of propagators. Note that the allowed ranges of $\beta+\rho$ and $\beta-\rho$ mean that the result is valid only when the Wilson lines are crossed.

Let us now study the most general case. We shall show that the four-point function corresponding to any arbitrary configuration of crossed Wilson lines has the same expression. Such a configuration, as depicted in figure 5 , is determined by four angles, namely $\alpha$, $\beta, \gamma$, and $\rho$. The four-point function is then

$$
\begin{aligned}
& \left\langle g_{R_{1}}^{-1}\left(0+\beta-\alpha, z_{1}\right) g_{R_{1}}\left(\pi-\beta-\alpha, z_{1}\right) \otimes g_{R_{2}}^{-1}\left(\pi / 2-\rho-\delta, z_{2}\right) g_{R_{2}}\left(3 \pi / 2+\rho-\delta, z_{2}\right)\right\rangle \\
= & \mathbb{1}+\frac{\hbar}{z_{1}-z_{2}} \frac{1}{2}\left(\widetilde{\Delta}_{\frac{\pi}{2}+\beta-\rho-\alpha+\delta}-\widetilde{\Delta}_{-\frac{\pi}{2}+\beta+\rho-\alpha+\delta}+\widetilde{\Delta}_{\frac{\pi}{2}-\beta+\rho-\alpha+\delta}-\widetilde{\Delta}_{-\frac{\pi}{2}-\beta-\rho-\alpha+\delta}\right) T_{R_{1}}^{a} \otimes T_{R_{2} a} \\
& +O\left(\hbar^{2}\right) .
\end{aligned}
$$

Here, to ensure single-valuedness of propagators, we require $-\frac{3 \pi}{2}<\beta-\rho-\alpha+\delta<\frac{\pi}{2}$, $-\frac{3 \pi}{2}<-\beta+\rho-\alpha+\delta<\frac{\pi}{2},-\frac{\pi}{2}<\beta+\rho-\alpha+\delta<\frac{3 \pi}{2}$ and $-\frac{\pi}{2}<-\beta-\rho-\alpha+\delta<$ $\frac{3 \pi}{2}$. However, to ensure that we are considering only crossed Wilson lines, we require the stronger conditions $-\frac{\pi}{2}<\beta-\rho-\alpha+\delta<\frac{\pi}{2},-\frac{\pi}{2}<-\beta+\rho-\alpha+\delta<\frac{\pi}{2},-\frac{\pi}{2}<\beta+\rho-\alpha+\delta<\frac{\pi}{2}$ and $-\frac{\pi}{2}<-\beta-\rho-\alpha+\delta<\frac{\pi}{2}$. These conditions in turn allow us to use (3.23), whereby we find that $(3.26)$ is

$$
\mathbb{1}+\frac{\hbar}{z_{1}-z_{2}} T_{R_{1}}^{a} \otimes T_{R_{2} a}+O\left(\hbar^{2}\right)
$$




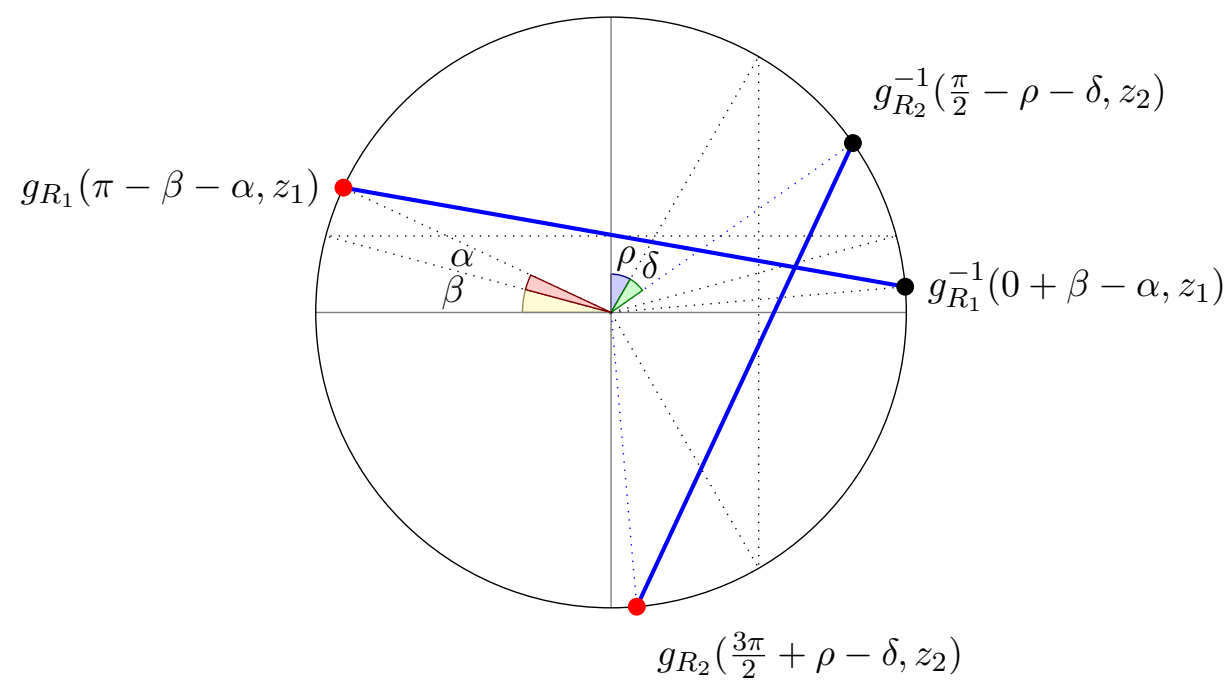

Figure 5. Arbitrarily inserted crossed Wilson lines.

We have thus shown that topological invariance along $\Sigma$ of the bulk correlation function of two crossed Wilson lines is reflected in the dual four-point function of the boundary $3 \mathrm{~d}$ "chiral" WZW model, at least up to order $\hbar$.

\subsection{Crossed Wilson lines at order $\hbar^{2}$}

Having found the expected order $\hbar$ contribution to the four-point function of arbitrarily inserted crossed Wilson lines in (3.26), we may improve on this and compute higher order contributions by using (free-field) $n$-point functions defined from the generating functional (3.5), for even $n$. We shall demonstrate this explicitly for the order $\hbar^{2}$ contribution to the correlation function of perpendicular Wilson lines.

Firstly, from the generating functional (3.5), the (free-field) four-point function

$$
\begin{aligned}
& \left\langle\phi^{a}(w) \phi^{b}(x) \phi^{c}(y) \phi^{d}(z)\right\rangle \\
= & \frac{\hbar^{2}}{4}\left(\Delta^{a b}(w-x) \Delta^{c d}(y-z)+\Delta^{a c}(w-y) \Delta^{b d}(x-z)+\Delta^{a d}(w-z) \Delta^{b c}(x-y)\right)
\end{aligned}
$$

can be found. Expanding the operators in the r.h.s. of (3.3) to quadratic order in $\phi$ as

$$
\begin{aligned}
g_{R_{1}}^{-1}\left(0, z_{1}\right) g_{R_{1}}\left(\pi, z_{1}\right)= & \mathbb{1}+\left(\phi_{a}\left(\pi, z_{1}\right)-\phi_{a}\left(0, z_{1}\right)\right) T_{R_{1}}^{a}+\left(-\phi_{a}\left(0, z_{1}\right) \phi_{b}\left(\pi, z_{1}\right)\right. \\
& \left.+\frac{1}{2} \phi_{a}\left(\pi, z_{1}\right) \phi_{b}\left(\pi, z_{1}\right)+\frac{1}{2} \phi_{a}\left(0, z_{1}\right) \phi_{b}\left(0, z_{1}\right)\right) T_{R_{1}}^{a} T_{R_{1}}^{b}+\ldots \\
g_{R_{2}}^{-1}\left(\pi / 2, z_{2}\right) g_{R_{2}}\left(3 \pi / 2, z_{2}\right)= & \mathbb{1}+\left(\phi_{a}\left(3 \pi / 2, z_{2}\right)-\phi_{a}\left(\pi / 2, z_{2}\right)\right) T_{R_{2}}^{a}+\left(-\phi_{a}\left(\pi / 2, z_{2}\right) \phi_{b}\left(3 \pi / 2, z_{2}\right)\right. \\
& \left.+\frac{1}{2} \phi_{a}\left(3 \pi / 2, z_{2}\right) \phi_{b}\left(3 \pi / 2, z_{2}\right)+\frac{1}{2} \phi_{a}\left(\pi / 2, z_{2}\right) \phi_{b}\left(\pi / 2, z_{2}\right)\right) T_{R_{2}}^{a} T_{R_{2}}^{b} \\
& +\ldots,
\end{aligned}
$$


we then find via (3.28) that, to order $\hbar^{2},(3.3)$ is

$$
\begin{aligned}
&\left\langle g_{R_{1}}^{-1}\left(0, z_{1}\right) g_{R_{1}}(\right.\left.\left.\pi, z_{1}\right) \otimes g_{R_{2}}^{-1}\left(\pi / 2, z_{2}\right) g_{R_{2}}\left(3 \pi / 2, z_{2}\right)\right\rangle \\
&=\mathbb{1}+\frac{\hbar}{z_{1}-z_{2}} T_{R_{1}}^{a} \otimes T_{R_{2} a} \\
&+\frac{\hbar^{2}}{4\left(z_{1}-z_{2}\right)^{2}}\left(\widetilde{\Delta}_{0-\frac{\pi}{2}}^{a c} \widetilde{\Delta}_{\pi-\frac{3 \pi}{2}}^{b d}+\widetilde{\Delta}_{2 \pi-\frac{3 \pi}{2}}^{a d} \widetilde{\Delta}_{\pi-\frac{\pi}{2}}^{b c}-\frac{1}{2}\left(\widetilde{\Delta}_{2 \pi-\frac{3 \pi}{2}}^{a c} \widetilde{\Delta}_{\pi-\frac{3 \pi}{2}}^{b d}+\widetilde{\Delta}_{2 \pi-\frac{3 \pi}{2}}^{a d} \widetilde{\Delta}_{\pi-\frac{3 \pi}{2}}^{b c}{ }^{2}\right)\right. \\
&-\frac{1}{2}\left(\widetilde{\Delta}_{0-\frac{\pi}{2}}^{a c} \widetilde{\Delta}_{\pi-\frac{\pi}{2}}^{b d}+\widetilde{\Delta}_{0-\frac{\pi}{2}}^{a d} \widetilde{\Delta}_{\pi-\frac{\pi}{2}}^{b c}\right)-\frac{1}{2}\left(\widetilde{\Delta}_{\pi-\frac{\pi}{2}}^{a c} \widetilde{\Delta}_{\pi-\frac{3 \pi}{2}}^{b d}+\widetilde{\Delta}_{\pi-\frac{3 \pi}{2}}^{a d} \widetilde{\Delta}_{\pi-\frac{\pi}{2}}^{b c}\right) \\
&-\frac{1}{2}\left(\widetilde{\Delta}_{0-\frac{\pi}{2}}^{a c} \widetilde{\Delta}_{2 \pi-\frac{3 \pi}{2}}^{b d}+\widetilde{\Delta}_{2 \pi-\frac{3 \pi}{2}}^{a d} \widetilde{\Delta}_{0-\frac{\pi}{2}}^{b c}\right)+\frac{1}{4}\left(\widetilde{\Delta}_{\pi-\frac{3 \pi}{2}}^{a c} \widetilde{\Delta}_{\pi-\frac{3 \pi}{2}}^{b d}+\widetilde{\Delta}_{\pi-\frac{3 \pi}{2}}^{a d} \widetilde{\Delta}_{\pi-\frac{3 \pi}{2}}^{b c}\right) \\
&+\frac{1}{4}\left(\widetilde{\Delta}_{\pi-\frac{\pi}{2}}^{a c} \widetilde{\Delta}_{\pi-\frac{\pi}{2}}^{b d}+\widetilde{\Delta}_{\pi-\frac{\pi}{2}}^{a d} \widetilde{\Delta}_{\pi-\frac{\pi}{2}}^{b c}\right)+\frac{1}{4}\left(\widetilde{\Delta}_{2 \pi-\frac{3 \pi}{2}}^{a c} \widetilde{\Delta}_{2 \pi-\frac{3 \pi}{2}}^{b d}+\widetilde{\Delta}_{2 \pi-\frac{3 \pi}{2}}^{a d} \widetilde{\Delta}_{2 \pi-\frac{3 \pi}{2}}^{b c}\right) \\
&\left.+\frac{1}{4}\left(\widetilde{\Delta}_{0-\frac{\pi}{2}}^{a c} \widetilde{\Delta}_{0-\frac{\pi}{2}}^{b d}+\widetilde{\Delta}_{0-\frac{\pi}{2}}^{a d} \widetilde{\Delta}_{0-\frac{\pi}{2}}^{b c}\right)\right) T_{R_{1}}^{a} T_{R_{1}}^{b} \otimes T_{R_{2}}^{c} T_{R_{2}}^{d}+\mathcal{O}\left(\hbar^{3}\right) \\
&=\mathbb{1}+\frac{\hbar}{z_{1}-z_{2}} T_{R_{1}}^{a} \otimes T_{R_{2} a}+\frac{\hbar^{2}}{4\left(z_{1}-z_{2}\right)^{2}}\left(T_{R_{1}}^{a} T_{R_{1}}^{b} \otimes T_{R_{2} a} T_{R_{2} b}+T_{R_{1}}^{a} T_{R_{1}}^{b} \otimes T_{R_{2} b} T_{R_{2} a}\right)+\mathcal{O}\left(\hbar^{3}\right),
\end{aligned}
$$

where we have used the notation $\widetilde{\Delta}_{\varphi}^{a b}=\widetilde{\Delta}_{\varphi} \delta^{a b}$ for brevity.

In a similar manner, one can compute contributions to the $R$-matrix of order $\hbar^{3}$ and above. Note that contributions at order $\hbar^{2}$ and above are not expected to remain invariant under moves of the local boundary operators that correspond to rotations and translations of the bulk Wilson lines, due to the framing anomaly that arises in the bulk theory at order $\hbar^{2}$ for non-perpendicular Wilson lines [3]. This framing anomaly ought to be computable in our boundary WZW model as well, by taking into account its interaction terms when computing correlation functions of local operators.

A slightly more involved calculation shows that the result of (3.30) holds, modulo the framing anomaly, for arbitrarily inserted Wilson lines (as depicted in figure 5), assuming the same constraints on the angles given below (3.26).

\subsection{Parallel Wilson lines}

The OPEs of parallel Wilson lines in 4d Chern-Simons theory do not have the same singular behaviour as correlation functions of crossed Wilson lines. In this subsection, we shall consider such correlation functions of parallel Wilson lines and show how they are captured by correlation functions of boundary operators. We shall focus on the free-field limit, at order $\hbar$, and retrieve the expected behaviour in this regime.

Using (3.2), the correlation function of the operators we are interested in (depicted in figure 6$)$ is

$$
\begin{aligned}
& \left\langle\mathcal{P} e^{\int_{\varphi=3 \pi / 2}^{\varphi=0} \mathcal{A}_{R_{1}}\left(z_{1}, \bar{z}_{1}\right)} \otimes \mathcal{P} e^{\int_{\varphi=\pi}^{\varphi=\pi / 2} \mathcal{A}_{R_{2}}\left(z_{2}, \bar{z}_{2}\right)}\right\rangle \\
= & \left\langle g_{R_{1}}^{-1}\left(0, z_{1}, \bar{z}_{1}\right) g_{R_{1}}\left(3 \pi / 2, z_{1}, \bar{z}_{1}\right) \otimes g_{R_{2}}^{-1}\left(\pi / 2, z_{2}, \bar{z}_{2}\right) g_{R_{2}}\left(\pi, z_{2}, \bar{z}_{2}\right)\right\rangle,
\end{aligned}
$$




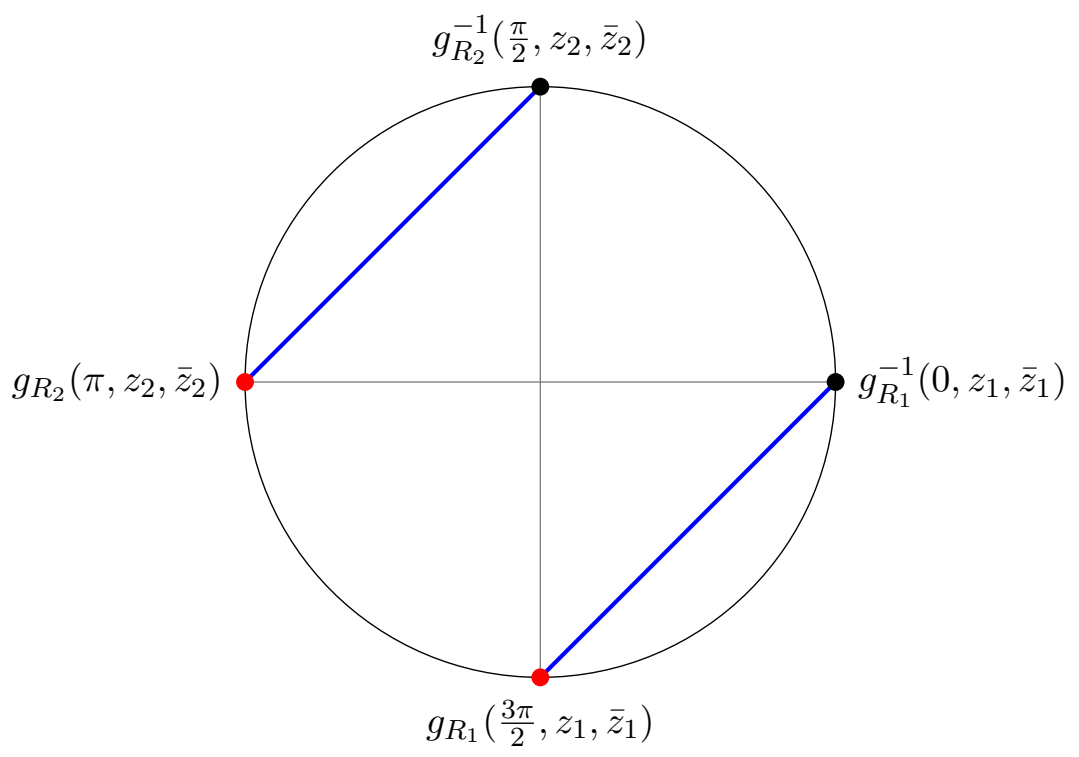

Figure 6. Parallel Wilson lines on $D$.

(note the difference from (3.3) in ordering of the boundary operators when $z_{1}=z_{2}$ and $\left.R_{1}=R_{2}\right)$.

Expanding each operator to linear order in $\phi$ and keeping only terms of quadratic or lower order in the fields (as in the discussion below (3.11)), we have

$$
\begin{aligned}
\mathbb{1} & \left\langle\phi_{a}\left(2 \pi, z_{1}\right) \phi_{c}\left(\pi / 2, z_{2}\right)\right\rangle T_{R_{1}}^{a} \otimes T_{R_{2}}^{c}-\left\langle\phi_{a}\left(2 \pi, z_{1}\right) \phi_{c}\left(\pi, z_{2}\right)\right\rangle T_{R_{1}}^{a} \otimes T_{R_{2}}^{c} \\
- & \left\langle\phi_{a}\left(3 \pi / 2, z_{1}\right) \phi_{c}\left(\pi / 2, z_{2}\right)\right\rangle T_{R_{1}}^{a} \otimes T_{R_{2}}^{c}+\left\langle\phi_{a}\left(3 \pi / 2, z_{1}\right) \phi_{c}\left(\pi, z_{2}\right)\right\rangle T_{R_{1}}^{a} \otimes T_{R_{2}}^{c}+O\left(\hbar^{2}\right) \\
= & \mathbb{1}-\frac{\hbar}{2} \delta_{a c} \frac{1}{z_{1}-z_{2}} \widetilde{\Delta}_{\frac{3 \pi}{2}} T_{R_{1}}^{a} \otimes T_{R_{2}}^{c}+\frac{\hbar}{2} \delta_{a c} \frac{1}{z_{1}-z_{2}} \widetilde{\Delta}_{\pi} T_{R_{1}}^{a} \otimes T_{R_{2}}^{c} \\
& +\frac{\hbar}{2} \delta_{a c} \frac{1}{z_{1}-z_{2}} \widetilde{\Delta}_{\pi} T_{R_{1}}^{a} \otimes T_{R_{2}}^{c}-\frac{\hbar}{2} \delta_{a c} \frac{1}{z_{1}-z_{2}} \widetilde{\Delta}_{\frac{\pi}{2}} T_{R_{1}}^{a} \otimes T_{R_{2}}^{c}+O\left(\hbar^{2}\right) \\
= & \mathbb{1}+O\left(\hbar^{2}\right),
\end{aligned}
$$

which is non-singular for $z_{1}=z_{2}$ at order $\hbar$, as expected. Here, we have used the previously derived fact that $\widetilde{\Delta}_{\frac{\pi}{2}}=\frac{1}{2}$, as well as $\widetilde{\Delta}_{\frac{3 \pi}{2}}=\frac{1}{2}$, which follows from (3.17), (3.19) and (3.20), and

$$
\begin{aligned}
\widetilde{\Delta}_{\pi} & =\frac{1}{2 \pi}\left(\sum_{k=1}^{\infty} \frac{(-1)^{k}}{i k}+\pi+\sum_{k=-\infty}^{-1} \frac{(-1)^{k}}{i k}\right) \\
& =\frac{1}{2 \pi}(i \ln 2+\pi-i \ln 2) \\
& =\frac{1}{2} .
\end{aligned}
$$

Also, note that we must define the propagator here with a different branch cut from that of previous sections, namely, from $r=0$ to $(r=R, \varphi=0)$. This effectively restricts $\varphi$ in (3.8) to take values in $(0,2 \pi)$. 


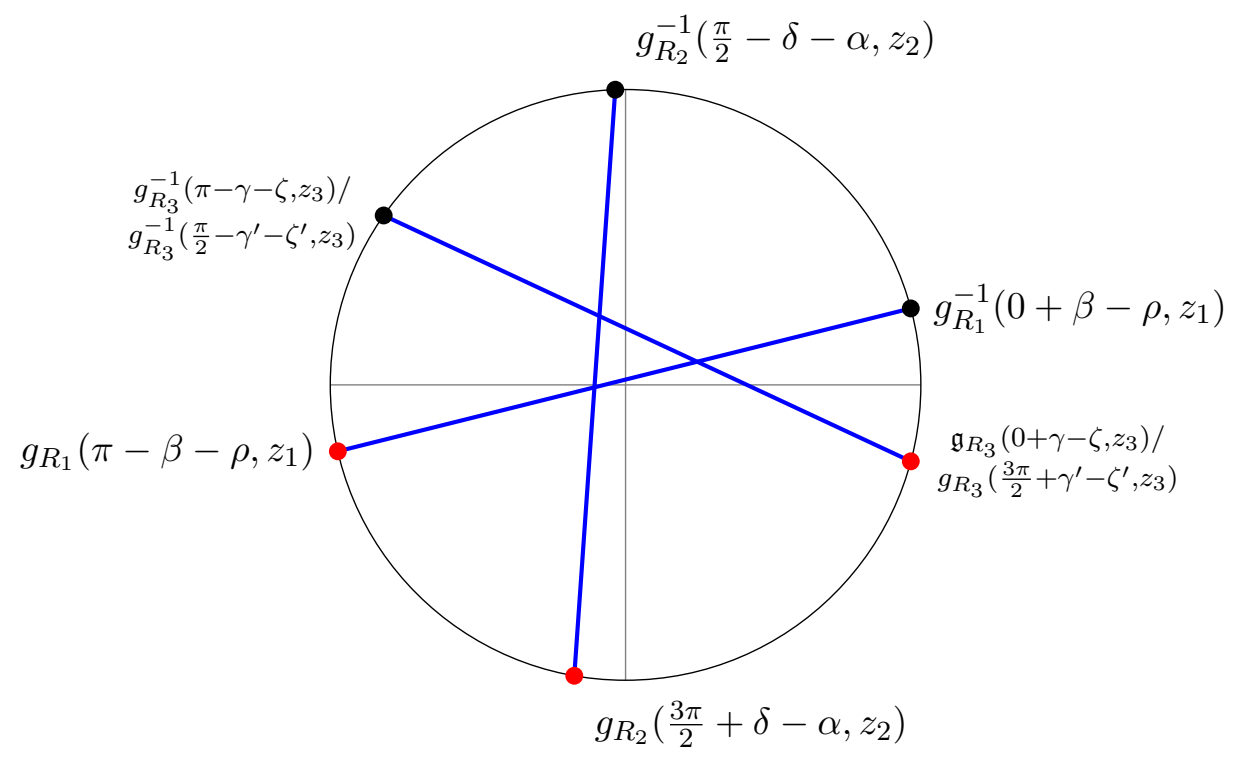

Figure 7. Three Wilson lines.

\subsection{Three Wilson lines}

We next consider correlation functions of three Wilson lines, all crossing each other but otherwise inserted arbitrarily (cf. figure 7), which corresponds to the following boundary correlator:

$$
\begin{aligned}
& \left\langle g_{R_{1}}^{-1}(0+\beta-\rho) g_{R_{1}}(\pi-\beta-\rho) \otimes g_{R_{2}}^{-1}\left(\frac{\pi}{2}-\delta-\alpha\right) g_{R_{2}}\left(\frac{3 \pi}{2}+\delta-\alpha\right)\right. \\
& \left.\quad \otimes g_{R_{3}}^{-1}(\pi-\gamma-\zeta) g_{R_{3}}(0+\gamma-\zeta)\right\rangle,
\end{aligned}
$$

where the dependence on $\mathbb{C}$ has been suppressed for brevity. Expanding each operator in (3.34) to linear order in $\phi$ we find

$$
\begin{aligned}
\mathbb{1} & +\left(\left\langle\phi_{a}\left(0+\beta+\rho, z_{1}\right) \phi_{c}\left(\pi / 2-\delta-\alpha, z_{2}\right)\right\rangle-\left\langle\phi_{a}\left(\pi-\beta-\rho, z_{1}\right) \phi_{c}\left(\pi / 2-\delta-\alpha, z_{2}\right)\right\rangle\right. \\
- & \left.\left\langle\phi_{a}\left(2 \pi+\beta-\rho, z_{1}\right) \phi_{c}\left(3 \pi / 2+\delta-\alpha, z_{2}\right)\right\rangle+\left\langle\phi_{a}\left(\pi-\beta-\rho, z_{1}\right) \phi_{c}\left(3 \pi / 2+\delta-\alpha, z_{2}\right)\right\rangle\right) T_{R_{1}}^{a} \otimes T_{R_{2}}^{c} \otimes \mathbb{1} \\
& +\left(\left\langle\phi_{a}\left(0+\beta-\rho, z_{1}\right) \phi_{c}\left(\pi / 2-\gamma^{\prime}-\zeta^{\prime}, z_{3}\right)\right\rangle-\left\langle\phi_{a}\left(2 \pi+\beta-\rho, z_{1}\right) \phi_{c}\left(3 \pi / 2+\gamma^{\prime}-\zeta^{\prime}, z_{3}\right)\right\rangle\right. \\
- & \left.\left\langle\phi_{a}\left(\pi-\beta-\rho, z_{1}\right) \phi_{c}\left(\pi / 2-\gamma^{\prime}-\zeta^{\prime}, z_{3}\right)\right\rangle+\left\langle\phi_{a}\left(\pi-\beta-\rho, z_{1}\right) \phi_{c}\left(3 \pi / 2+\gamma^{\prime}-\zeta^{\prime}, z_{3}\right)\right\rangle\right) T_{R_{1}}^{a} \otimes \mathbb{1} \otimes T_{R_{3}}^{c} \\
+ & \left(\left\langle\phi_{a}\left(\pi / 2-\delta-\alpha, z_{2}\right) \phi_{c}\left(\pi-\gamma-\zeta, z_{3}\right)\right\rangle-\left\langle\phi_{a}\left(\pi / 2-\delta-\alpha, z_{2}\right) \phi_{c}\left(0+\gamma-\zeta, z_{3}\right)\right\rangle\right. \\
- & \left.\left\langle\phi_{a}\left(3 \pi / 2+\delta-\alpha, z_{2}\right) \phi_{c}\left(\pi-\gamma-\zeta, z_{3}\right)\right\rangle+\left\langle\phi_{a}\left(3 \pi / 2+\delta-\alpha, z_{2}\right) \phi_{c}\left(2 \pi+\gamma-\zeta, z_{3}\right)\right\rangle\right) \mathbb{1} \otimes T_{R_{2}}^{a} \otimes T_{R_{3}}^{c} \\
+ & O\left(\hbar^{2}\right) \\
= & \mathbb{1}+\frac{\hbar}{z_{1}-z_{2}} \frac{1}{2}\left(\frac{1}{2}+\frac{1}{2}+\frac{1}{2}+\frac{1}{2}\right) T_{R_{1}}^{a} \otimes T_{R_{2} a} \otimes \mathbb{1}+\frac{\hbar}{z_{1}-z_{3}} \frac{1}{2}\left(\frac{1}{2}+\frac{1}{2}+\frac{1}{2}+\frac{1}{2}\right) T_{R_{1}}^{a} \otimes \mathbb{1} \otimes T_{R_{3} a} \\
& -\frac{\hbar}{z_{3}-z_{2}} \frac{1}{2}\left(\frac{1}{2}+\frac{1}{2}+\frac{1}{2}+\frac{1}{2}\right) \mathbb{1} \otimes T_{R_{2}}^{a} \otimes T_{R_{3} a}+O\left(\hbar^{2}\right) \\
= & \mathbb{1}+\frac{\hbar}{z_{1}-z_{2}} T_{R_{1}}^{a} \otimes T_{R_{2} a} \otimes \mathbb{1}+\frac{\hbar}{z_{1}-z_{3}} T_{R_{1}}^{a} \otimes \mathbb{1} \otimes T_{R_{3} a}+\frac{\hbar}{z_{2}-z_{3}} \mathbb{1} \otimes T_{R_{2}}^{a} \otimes T_{R_{3} a}+O\left(\hbar^{2}\right),
\end{aligned}
$$


where various constraints on the angles are necessary for single-valuedness of propagators and to ensure that the Wilson lines are all crossed. Once again, there is agreement with the bulk $4 \mathrm{~d}$ Chern-Simons computation.

We may further compute the correlation function (3.34) to order $\hbar^{2}$ by expanding each operator in (3.34) to quadratic order in $\phi$. Doing so, we find the following expression (modulo the framing anomaly):

$$
\begin{aligned}
& \mathbb{1}+\frac{\hbar}{z_{1}-z_{2}} T_{R_{1}}^{a} \otimes T_{R_{2} a} \otimes \mathbb{1}+\frac{\hbar}{z_{1}-z_{3}} T_{R_{1}}^{a} \otimes \mathbb{1} \otimes T_{R_{3} a}+\frac{\hbar}{z_{2}-z_{3}} \mathbb{1} \otimes T_{R_{2}}^{a} \otimes T_{R_{3} a} \\
& +\frac{\hbar^{2}}{4\left(z_{1}-z_{2}\right)^{2}}\left(T_{R_{1}}^{a} T_{R_{1}}^{b} \otimes T_{R_{2} a} T_{R_{2} b} \otimes \mathbb{1}+T_{R_{1}}^{a} T_{R_{1}}^{b} \otimes T_{R_{2} b} T_{R_{2} a} \otimes \mathbb{1}\right) \\
& +\frac{\hbar^{2}}{4\left(z_{1}-z_{3}\right)^{2}}\left(T_{R_{1}}^{a} T_{R_{1}}^{b} \otimes \mathbb{1} \otimes T_{R_{3} a} T_{R_{3} b}+T_{R_{1}}^{a} T_{R_{1}}^{b} \otimes \mathbb{1} \otimes T_{R_{3} b} T_{R_{3} a}\right) \\
& +\frac{\hbar^{2}}{4\left(z_{2}-z_{3}\right)^{2}}\left(\mathbb{1} \otimes T_{R_{2}}^{a} T_{R_{2}}^{b} \otimes T_{R_{3} a} T_{R_{3} b}+\mathbb{1} \otimes T_{R_{1}}^{a} T_{R_{1}}^{b} \otimes T_{R_{2} b} T_{R_{2} a}\right) \\
& +\frac{\hbar^{2}}{2\left(z_{1}-z_{2}\right)\left(z_{1}-z_{3}\right)}\left(T_{R_{1}}^{a} T_{R_{1}}^{b} \otimes T_{R_{2} a} \otimes T_{R_{3} b}+T_{R_{1}}^{a} T_{R_{1}}^{b} \otimes T_{R_{2} b} \otimes T_{R_{3} a}\right) \\
& +\frac{\hbar^{2}}{2\left(z_{1}-z_{2}\right)\left(z_{2}-z_{3}\right)}\left(T_{R_{1}}^{a} \otimes T_{R_{2} a} T_{R_{2} b} \otimes T_{R_{3}}^{b}+T_{R_{1}}^{a} \otimes T_{R_{2}}^{b} T_{R_{2} a} \otimes T_{R_{3} b}\right) \\
& +\frac{\hbar^{2}}{2\left(z_{1}-z_{3}\right)\left(z_{2}-z_{3}\right)}\left(T_{R_{1}}^{a} \otimes T_{R_{2}}^{b} \otimes T_{R_{3} a} T_{R_{3} b}+T_{R_{1}}^{a} \otimes T_{R_{2}}^{b} \otimes T_{R_{3} b} T_{R_{3} a}\right)+O\left(\hbar^{3}\right) .
\end{aligned}
$$

This result agrees with the bulk $4 \mathrm{~d}$ Chern-Simons computation. To see this, let us consider the configurations in figure 8. From the bulk theory, we know that the equivalence of these two configurations gives rise to the Yang-Baxter equation

$$
\widetilde{R}_{12} \widetilde{R}_{13} \widetilde{R}_{23}=\widetilde{R}_{23} \widetilde{R}_{13} \widetilde{R}_{12}
$$

where

$$
\begin{aligned}
\widetilde{R}_{12}= & \mathbb{1}+\frac{\hbar}{z_{1}-z_{2}} T_{R_{1}}^{a} \otimes T_{R_{2} a} \otimes \mathbb{1} \\
& +\frac{\hbar^{2}}{4\left(z_{1}-z_{2}\right)^{2}}\left(T_{R_{1}}^{a} T_{R_{1}}^{b} \otimes T_{R_{2} a} T_{R_{2} b} \otimes \mathbb{1}+T_{R_{1}}^{a} T_{R_{1}}^{b} \otimes T_{R_{2} b} T_{R_{2} a} \otimes \mathbb{1}\right)+\mathcal{O}\left(\hbar^{3}\right), \\
\widetilde{R}_{13}= & \mathbb{1}+\frac{\hbar}{z_{1}-z_{3}} T_{R_{1}}^{a} \otimes \mathbb{1} \otimes T_{R_{3} a} \\
& +\frac{\hbar^{2}}{4\left(z_{1}-z_{3}\right)^{2}}\left(T_{R_{1}}^{a} T_{R_{1}}^{b} \otimes \mathbb{1} \otimes T_{R_{3} a} T_{R_{3} b}+T_{R_{1}}^{a} T_{R_{1}}^{b} \otimes \mathbb{1} \otimes T_{R_{3} b} T_{R_{3} a}\right)+\mathcal{O}\left(\hbar^{3}\right), \\
\widetilde{R}_{23}=\mathbb{1} & +\frac{\hbar}{z_{2}-z_{3}} \mathbb{1} \otimes T_{R_{2}}^{a} \otimes T_{R_{3} a} \\
& +\frac{\hbar^{2}}{4\left(z_{2}-z_{3}\right)^{2}}\left(\mathbb{1} \otimes T_{R_{2}}^{a} T_{R_{2}}^{b} \otimes T_{R_{3} a} T_{R_{3} b}+\mathbb{1} \otimes T_{R_{2}}^{a} T_{R_{2}}^{b} \otimes T_{R_{3} b} T_{R_{3} a}\right)+\mathcal{O}\left(\hbar^{3}\right) .
\end{aligned}
$$

We shall now make use of the identity

$$
\frac{\left[T_{R_{1}}^{a}, T_{R_{1}}^{b}\right] \otimes T_{R_{2} a} \otimes T_{R_{3} b}}{\left(z_{1}-z_{2}\right)\left(z_{1}-z_{3}\right)}+\frac{T_{R_{1}}^{a} \otimes\left[T_{R_{2} a}, T_{R_{2}}^{b}\right] \otimes T_{R_{3} b}}{\left(z_{1}-z_{2}\right)\left(z_{2}-z_{3}\right)}+\frac{T_{R_{1}}^{a} \otimes T_{R_{2}}^{b} \otimes\left[T_{R_{3} a}, T_{R_{3} b}\right]}{\left(z_{1}-z_{3}\right)\left(z_{2}-z_{3}\right)}=0
$$




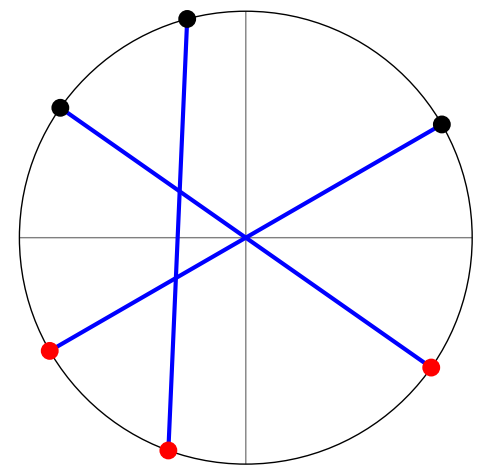

(a)

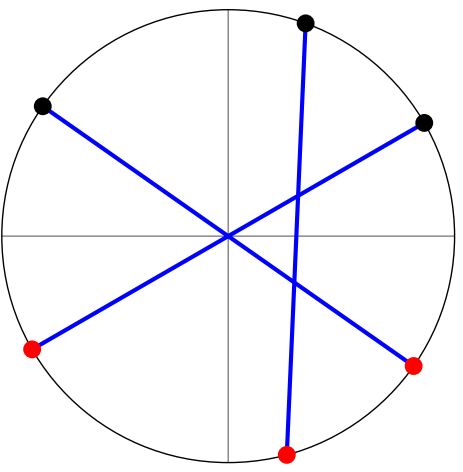

(b)

Figure 8. The Yang-Baxter equation is realized by moving a Wilson line across the intersection of two other Wilson lines.

which follows since the classical $r$-matrix, $r_{i j}=\frac{T_{R_{i}}^{a} \otimes T_{R_{j} a}}{z_{i}-z_{j}}(i, j=1,2,3$, where $j>i)$, obeys the classical Yang-Baxter equation. Using (3.39), the last three terms at order $\hbar^{2}$ of (3.36) can be shown to be

$$
\begin{aligned}
& \frac{\hbar^{2}}{\left(z_{1}-z_{2}\right)\left(z_{1}-z_{3}\right)} T_{R_{1}}^{a} T_{R_{1}}^{b} \otimes T_{R_{2} a} \otimes T_{R_{3} b}+\frac{\hbar^{2}}{\left(z_{1}-z_{2}\right)\left(z_{2}-z_{3}\right)} T_{R_{1}}^{a} \otimes T_{R_{2} a} T_{R_{2} b} \otimes T_{R_{3}}^{b} \\
& +\frac{\hbar^{2}}{\left(z_{1}-z_{3}\right)\left(z_{2}-z_{3}\right)} T_{R_{1}}^{a} \otimes T_{R_{2}}^{b} \otimes T_{R_{3} a} T_{R_{3} b},
\end{aligned}
$$

whereby (3.36) agrees with the 1.h.s. of (3.37). Alternatively, we can use (3.39) such that the last three terms at order $\hbar^{2}$ of (3.36) are

$$
\begin{aligned}
& \frac{\hbar^{2}}{\left(z_{1}-z_{2}\right)\left(z_{1}-z_{3}\right)} T_{R_{1}}^{a} T_{R_{1}}^{b} \otimes T_{R_{2} b} \otimes T_{R_{3} a}+\frac{\hbar^{2}}{\left(z_{1}-z_{2}\right)\left(z_{2}-z_{3}\right)} T_{R_{1}}^{a} \otimes T_{R_{2}}^{b} T_{R_{2} a} \otimes T_{R_{3} b} \\
& +\frac{\hbar^{2}}{\left(z_{1}-z_{3}\right)\left(z_{2}-z_{3}\right)} T_{R_{1}}^{a} \otimes T_{R_{2}}^{b} \otimes T_{R_{3} b} T_{R_{3} a},
\end{aligned}
$$

whereby (3.36) agrees with the r.h.s. of (3.37). Thus, the boundary six-point function (3.36) is in agreement with the bulk correlation function of three Wilson lines up to order $\hbar^{2}$, modulo the framing anomaly. We expect that this will hold at higher orders of $\hbar$ as well.

\section{Acknowledgments}

We would like to thank Benjo Fraser, Lennart Schmidt, Meng-Chwan Tan, Junya Yagi, and Masahito Yamazaki for helpful discussions and suggestions, and the anonymous referee for helpful comments. Results in this paper were presented at Kavli IPMU in May, 2019, and we thank the audience of this talk for feedback. We would also like to thank Kavli IPMU for hospitality. This work is supported by the MOE Tier 2 grant R-144-000-396-112.

Open Access. This article is distributed under the terms of the Creative Commons Attribution License (CC-BY 4.0), which permits any use, distribution and reproduction in any medium, provided the original author(s) and source are credited. 


\section{References}

[1] K. Costello, Integrable lattice models from four-dimensional field theories, Proc. Symp. Pure Math. 88 (2014) 3 [arXiv:1308.0370] [INSPIRE].

[2] K. Costello, Supersymmetric gauge theory and the Yangian, arXiv:1303.2632 [INSPIRE].

[3] K. Costello, E. Witten and M. Yamazaki, Gauge Theory and Integrability, I, ICCM Not. 06 (2018) 46 [arXiv:1709.09993] [InSPIRE].

[4] K. Costello, E. Witten and M. Yamazaki, Gauge Theory and Integrability, II, ICCM Not. 06 (2018) 120 [arXiv: 1802.01579] [INSPIRE].

[5] K. Costello and M. Yamazaki, Gauge Theory And Integrability, III, arXiv:1908.02289 [INSPIRE].

[6] R. Bittleston and D. Skinner, Gauge Theory and Boundary Integrability, JHEP 05 (2019) 195 [arXiv: 1903.03601] [INSPIRE].

[7] R. Bittleston and D. Skinner, Gauge theory and boundary integrability. Part II. Elliptic and trigonometric cases, JHEP 06 (2020) 080 [arXiv: 1912.13441] [INSPIRE].

[8] M. Yamazaki, New T-duality for Chern-Simons Theory, JHEP 12 (2019) 090 [arXiv: 1904.04976] [INSPIRE].

[9] V.G. Drinfeld, Quantum Groups, in proceedings of the International Congress of Mathematicians, Berkeley, 1986, American Mathematical Society (1987).

[10] V. Chari and A. Pressley, A Guide to Quantum Groups, Cambridge University Press (1994).

[11] S. Elitzur, G.W. Moore, A. Schwimmer and N. Seiberg, Remarks on the Canonical Quantization of the Chern-Simons-Witten Theory, Nucl. Phys. B 326 (1989) 108 [InSPIRE].

[12] E. Witten, Nonabelian Bosonization in Two-Dimensions, Commun. Math. Phys. 92 (1984) 455 [INSPIRE].

[13] S.K. Donaldson, Anti-self-dual Yang-Mills connections over complex algebraic surfaces and stable vector bundles, Proc. London Math. Soc. 3 (1985) 1.

[14] V.P. Nair and J. Schiff, A Kähler-\{Chern-Simons\} Theory and Quantization of Instanton Moduli Spaces, Phys. Lett. B 246 (1990) 423 [INSPIRE].

[15] A. Losev, G.W. Moore, N. Nekrasov and S. Shatashvili, Four-dimensional avatars of two-dimensional RCFT, Nucl. Phys. B Proc. Suppl. 46 (1996) 130 [hep-th/9509151] [INSPIRE].

[16] T. Inami, H. Kanno, T. Ueno and C.-S. Xiong, Two toroidal Lie algebra as current algebra of four-dimensional Kähler WZW model, Phys. Lett. B 399 (1997) 97 [hep-th/9610187] [INSPIRE].

[17] E. Witten, Integrable Lattice Models From Gauge Theory, Adv. Theor. Math. Phys. 21 (2017) 1819 [arXiv:1611.00592] [INSPIRE].

[18] S.V. Ketov, All loop finiteness of the four-dimensional Donaldson-Nair-Schiff nonlinear sigma model, Phys. Lett. B 383 (1996) 390 [hep-th/9604141] [INSPIRE].

[19] F. Delduc, S. Lacroix, M. Magro and B. Vicedo, A unifying $2 d$ action for integrable $\sigma$-models from 4d Chern-Simons theory, arXiv:1909.13824 [INSPIRE].

[20] B. Vicedo, Holomorphic Chern-Simons theory and affine Gaudin models, arXiv:1908.07511 [INSPIRE]. 